\title{
A preliminary overview of the Brazilian Apioninae (Coleoptera: Brentidae) with an illustrated key for genera, and a checklist with distribution information
}

\author{
Wesley Oliveira de Sousa ${ }^{1 *}{ }^{\circledR}$, Cibele Stramare Ribeiro-Costa ${ }^{2}{ }^{\circledR}$, Germano Henrique Rosado-Neto ${ }^{3}$ \\ ${ }^{1}$ Universidade Federal de Mato Grosso, Departamento de Ciências Biológicas, Campus Universitário de \\ Rondonópolis, Rodovia Rondonópolis-Guiratinga MT 270, Km 6, s/n, Rondonópolis, MT, Brasil \\ ${ }^{2}$ Universidade Federal do Paraná, Departamento de Zoologia, Laboratório de Sistemática e Bioecologia de \\ Coleoptera, Centro Politécnico s/n, Jardim das Américas, 81531-980, Curitiba, PR, Brasil \\ ${ }^{3}$ Universidade Federal do Paraná, Setor de Ciências Biológicas, Departamento de Zoologia, Centro \\ Politécnico s/n, Jardim das Américas, 81531-980, Curitiba, PR, Brasil \\ *Corresponding author: Wesley Oliveira de Sousa, e-mail: entomoi@hotmail.com
}

DE SOUSA, W. O., RIBEIRO-COSTA, C. S., ROSADO-NETO, G. H. A preliminary overview of the Brazilian Apioninae (Coleoptera: Brentidae) with an illustrated key for genera, and a checklist with distribution information. Biota Neotropica. 19(4): e20190813. http://dx.doi.org/10.1590/1676-0611-BN-2019-0813

\begin{abstract}
Here we examine the taxonomic diversity of the Brazilian Apioninae (Coleoptera: Brentidae) and provide an update based on the literature and through examination of material in primary Brazilian collections. Ten genera and 89 species are valid and we include 30 ( 25 genera, 5 species $)$ new distribution records. Chrysapion Kissinger, 1968 is first reported in Brazil, restricted to the northeast. Ranges now include the Amazon (three genera and one subgenus) and Atlantic (four genera and one subgenus) forests, the Pantanal (five genera) and Cerrado (three genera). The ranges of several genera now include more than one new biome. An identification key (including images of adults and illustrations of male genitalia) also includes the 10 Brazilian genera of Apioninae for the first time.

Keywords: Curculionoidea, Neotropical Region, taxonomy, systematic.
\end{abstract}

\section{Uma visão geral preliminar dos Apioninae (Coleoptera: Brentidae) brasileiros: chave ilustrada para identificação de gêneros, lista de espécies e registros de distribuição}

Resumo: O conhecimento sobre a diversidade taxonômica da fauna de Apioninae (Coleoptera: Brentidae) do Brasil é atualizado com base na literatura disponível e exame de material depositado nas principais coleções públicas do país. Dez gêneros e de 89 espécies válidas foram incluídas em 30 novos registros de distribuição (25 gêneros e cinco espécies). Chrysapion Kissinger, 1968 é registrado pela primeira para o Brasil, restrito aos biomas do Nordeste do Brasil. A distribuição geográfica inclui Amazônia (três gêneros e um subgênero) e Mata Atlântica (quatro gêneros e um subgênero), o Pantanal (cinco gêneros) e Cerrado (três gêneros). A distribuição de vários gêneros é atualizada para mais de um bioma. Uma chave de identificação (com imagens dos adultos e desenhos da genitália masculina) é apresentada pela primeira vez para os 10 gêneros de Apioninae que ocorrem no Brasil.

Palavras-chave: Curculionoidea, Região Neotropical, sistemática, taxonomia. 


\section{Introduction}

The subfamily Apioninae (Brentidae sensu latu) comprises a monophyletic group (Alonso-Zarazaga \& Wanat 2014; Marvaldi et al. 2002; Kuschel 1995; Wanat 2001) of very small beetles $(0.75-13.00 \mathrm{~mm}$ in length, $>95 \%<3 \mathrm{~mm})$. This subfamily differs from the other Curculionoidea by the following: a markedly convex to globose, pear-shaped (Apion is Greek for 'small pear') body (except Antliarhinitae); straight antennae with a compact club that appear geniculate in some Rhadinocybini; short, two-segmented, palps; nine-striate elytra (Apionini) with some genera having remainders of a tenth; brentid-type abdomen; two large, usually convex, first ventrites, the second almost vertical at apex; the last three ventrites small, forming a valve-like structure that opens to extrude genitalia; usually long, cylindrical trochanters but which may be short in several basal lineages; tegmen with fully developed and diverse parameral plate (Alonso-Zarazaga \& Wanat 2014; Anderson \& Kissinger 2002; Jones et al. 2012; Kissinger 2005a; Ptaszynska et al. 2012).

Adults feed on living plant tissues, including all green parts as well as fruit. Larvae are usually endophagous and also feed on different plant parts, including stems, roots, inflorescences, fruit, seeds and tissues forming gall-like structures. In contrast to Arctic fauna of both the Old and New Worlds, the fauna of the Neotropical Region is very poorly known (Alonso-Zarazaga \& Wanat 2014). While some few basal lineages of the Apioninae are associated with gymnosperms (Kissinger 1968; Alonso-Zarazaga and Wanat 2014), most basal groups are associated with plants in the clade Magnoliid as hosts (Alonso-Zarazaga \& Wanat 2014). More apical apionines (Apionitae: Apionini) have at least 23 families in 15 orders of dicot angiosperms (Adoxaceae, Apiaceae, Asteraceae, Polygonaceae, Caprifoliaceae, Ebenaceae, Euphorbiaceae, Fabaceae, Lamiaceae, Malvaceae, Melastomataceae, Proteaceae, Rhamnaceae, Rutaceae, Salicaceae and Verbenaceae; Alonso-Zarazaga \& Wanat 2014; Anderson \& Kissinger 2002; Badenes-Perez \& Jhonson 2007; Lima et al. 2008; Maia 2012). However, host associations of the Apioninae in South America are largely unknown (Alonso-Zarazaga \& Wanat 2014).

More than 2,200 species of Apioninae have been described (Alonso-Zarazaga \& Wanat, 2014), where they are found from high altitudes to sea level throughout the region (Alonso-Zarazaga 2004; Anderson \& Kissinger 2002; Oberprieler et al. 2007; Alonso-Zarazaga $\&$ Wanat 2014) and where the species comprise about 205 genera and subgenera (Alonso-Zarazaga \& Wanat 2014), seven supertribes and nine tribes (Bouchard et al. 2011; Alonso-Zarazaga 1990).

Kissinger (1968) pioneered the study of the genera and subgenera of Apion while focusing on the fauna of North and Central America, where he described an extraordinary diversity of forms and demonstrated that the tegmen of male genitalia provides important characters to determine their taxonomy. In South America, the Apioninae comprise 190 species (Wibmer \& O'Brien 1986) in 19 genera, 10 of which were thought to be endemic (Kissinger 1968, 2002, 2003, 2005a, b). Among other South American countries, Brazil also has no recent studies of the Apioninae and the little taxonomic information available can be found in 17 published studies that include 86 species (summarized and listed under the genus Apion Herbst, 1797 in the catalog of Wibmer and O'Brien 1986).

Some subgenera of Apion were elevated to genera after Kissinger (1968) and Wibmer and O'Brien (1986) laid the groundwork (Alonso-Zarazaga 1990; Kissinger 1992). Subsequently, new genera and subgenera were proposed (Alonso-Zarazaga 1990; Kissinger 1990, 1991, 1998, 1999b, 2002, 2005a). In the catalog of the families and genera of Curculionoidea (Alonso-Zarazaga \&Lyal 1999), only five genera in the Apioninae were reported in Brazil, each of which only incluced the type species: Neapion Alonso-Zarazaga, 1990 (Ixapiini), Apionion Kissinger, 1998, Bothryopteron Wagner, 1912a, Coelopterapion Wagner 1912a and Stenapion Wagner 1912a.

Currently, the only classification for suprageneric and generic Apioninae taxa is based on Palearctic fauna (Alonso-Zarazaga 1990, Ptaszynska et al. 2012, Winter et al. 2017). The Alonso-Zarazaga classification scheme does not include many North and Central American and most South American taxa, nor do the molecular studies of Ptaszynska et al. (2012) and Winter et al. (2017) include North, Central and South American taxa. Consequently, the basis for identification of the Apioninae of South America continues to be Kissinger (1968), even though obsolete. The genus Apion is now considered to be a monophyletic genus of 16 species that is restricted to the Palearctic (Alonso-Zarazaga 1990) and so many species listed by Kissinger (1968) remain in incorrect genera.

Today, hundreds of specimens have accumulated in collections in South American institutions under the genus Apion. This material is very important for taxonomic and systematic understanding of the Apioninae. Thus, we present a first step in understanding the Apioninae of Brazil by providing a checklist of genera and species, with their geographic distributions, host plants, citations of the relevant literature, and a key for the genera including photographs and illustrations.

\section{Material and Methods}

Specimens of the Apioninae were examined between August 2016 and March 2017 at the following institutions: Coleção Entomológica Pe. J. S. Moure, Departamento de Zoologia, Universidade Federal do Paraná, Curitiba (DZUP); Coleção Entomológica do Instituto de Biociências, Universidade Federal de Mato Grosso, Cuiabá (UFMT); Instituto Nacional de Pesquisas da Amazônia, Manaus (INPA); Laboratório de Ecologia e Taxonomia de Artrópodes Terrestres, Universidade Federal de Mato Grosso, Cuiabá (LETA); Museu Nacional, Universidade Federal do Rio de Janeiro, Rio de Janeiro (MNRJ); Museu de Zoologia, Universidade de São Paulo, São Paulo (MZSP).

Taxa identification, development of the dichotomous key and the species checklist of Brazilian Apioninae were based on Wagner (1910, 1912a, b, c, 1914, 1915), Kissinger (1968, 1998, 2002, 2003, 2005a, b), O'Brien \& Wibmer (1982), Wibmer \& O’Brien (1986) and Alonso-Zarazaga \& Lyal (1999). We adapted and modified the genera identification key from Alonso-Zarazaga (2004). We based the identification key on male characteristics because they are relevant for specific, generic and suprageneric classifications and diagnoses (Anderson \& Kissinger 2002; Wanat et al. 2001). To better examine the sexes and genitalia morphology, dried specimens were softened in lukewarm water with detergent for 24 hours. Genitalia extraction methods follow Alonso-Zarazaga (1989) and terminology for external morphology follows Alonso-Zarazaga \& Wanat (2014).

We examined the type material of the following 13 species: Apion cydoniae Bondar, 1950 and A. zikani Heller, 1922 (Museu Nacional, Universidade Federal do Rio de Janeiro, Rio de Janeiro, Brazil MNRJ); Apion argentinum Béguin-Billecocq 1909, A. contrarium 
Béguin-Billecocq, 1909, A desbordesi Béguin-Billecocq, 1911, A. ingratum Béguin-Billecocq, 1909, A. lativentre Béguin-Billecocq, 1909, A. salpingoides Béguin-Billecocq, 1909, A. simplex Béguin-Billecocq, 1909, A. rufonigrum Béguin-Billecocq, 1911 and A. tucumanense BéguinBillecocq, 1909 (Museu Argentino de Ciencias Naturales "Bernardino Rivadavia", Buenos Aires, Argentina - MACN); Neapion (Neotropion) diringsi De Sousa \& Ribeiro-Costa, 2018 (MZSP) and Neapion (Neotropion) marquesae De Sousa \& Ribeiro-Costa, 2018 (DZUP).

We examined photographic images of the type material of the following species: Apion areolatum Kirsch, 1874, A. luteipes Kirsch, 1874 and A. opacum Kirsch, 1874 deposited in the Staatliches Museum für Tierkunde, Dresden (SMTD); A. novaeteutoniae Voss, 1959b, A. phaseoli Voss, 1958 and A. sesbaniae Voss, 1958 (Zoological Museum, Hamburg); Apion clarki Kissinger, 1990 (Smithsonian National Museum of Natural History, Washington, USA - USMN); Apion hibisci Fall, 1918, A. neolentum Kissinger, 1968, A. umboniferum Fall, 1898, A. xanthoxyli Fall, 1898 (Database of Zoological Colletions - CVZBASE of the Museum of Comparative Zoology - Harvard University, http://mczbase. mcz.harvard.edu/name/Apion). We also examined photographic images and redescriptions in Kissinger (1968) of the following species: Apion americanum Wagner, 1908, Apion aurichalceum Wagner, 1912b, Apion bryanti Wagner, 1914, Apion constricticolle Sharp, 1890, Apion chiriquense Sharp, 1890, Apion crassum Fall, 1898, Apion grallarium Sharp, 1891a, A. peculiare Wagner, 1909, A. spinitarse Wagner, 1912a and $A$. spretissimum Sharp, 1890. We examined photographic images of material named Chrysapion auctum (Sharp, 1890) and Chrysapion tantillum (Sharp, 1890) from Arizona State University Hasbrouck Insect Collection (http://symbiota4.acis.ufl.edu/scan/portal/). Some species are very conspicuous, well-illustrated or labeled in such a way as to make examination of the type material unnecessary (e.g. Apion binodosum Wagner, 1912a, A. cionoides Wagner, 1912a, A. testaceum Wagner, 1912a., A. luteirostre Gerstaecker, 1854, A. brevicorne Gerstaecker 1854, A. ensirostre Gerstaecker, 1854, A. periscelis Gyllenhal, 1839, A. pseudampullula Voss, 1940, A. undulipenne Wagner, 1912a).

We indicate new records in the geographic distribution of the checklist by placing them in parentheses. Geographical records from the literature were complemented by the examination of the labels of the specimens deposited in the Brazilian public institution collections.

Classification for the suprageneric taxa is in alphabetical order in the checklist, following Bouchard et al. (2011). Genera and species determined to be Incertae sedis are placed in a special section at the end of the checklist.

Suprageneric group names are typed in bold capital letters, while genera, subgenera and species group names are both in italic and bold capitals, followed by author, date and page of publication preceded by a colon (:). All genera and subgenera include: type-species, synonyms, distribution in bold type face, country in parentheses, host plants, references and page of publication preceded by an colon (:), remarks explaining how the taxa was recognized. Genera are left-justified while subgenera are indented and in parentheses. The most current species name is followed by author, date and page of publication in parentheses. We include synonyms in chronological order, followed by comments (if needed, in parentheses) and references between square brackets; distribution in bold type faces and states between in parentheses, references and page of publication, remarks about the species and material examined when studied.
Photographic images were taken using a Leica MZ16 stereomicroscope, Leica DFC 500 camera, Leica 3D viewer module and version montage 4.7 (software). Drawings and images were edited in Photoshop CS6. Schematics of the male genitalia were drawn using a stereomicroscope or a microscope with a light camera coupled system or image capture system.

\section{Results}

From ca 230 specimens in collections in Brazil, we identified 10 genera and two subgenera of Apioninae in a total of 43 species. We identify 25 Brazilian species, 15 of which are placed in "Apion" in the broadest sense. Thirty new records are included, one for Brazil: Chrysapion Kissinger, 1968. Another 24 records are new for Brazil: the geographic distribution of Neapion Alonso-Zarazaga, 1990, subgenus Neotropion Alonso-Zarazaga, 1990 is extended to include Amazon (Acre and Pará) and Atlantic (São Paulo and Paraná) forests; Coelocephalapion Wagner, 1914 is extended to the Pantanal (Mato Grosso and Mato Grosso do Sul); Apionion Kissinger, 1998 now includes the Pantanal (Mato Grosso) and Atlantic Forest (Rio de Janeiro); Bothryopteron Wagner, 1912 is extended to the Amazon (Maranhão), Pantanal (Mato Grosso), Cerrado (Tocantins and Goiás) and Atlantic Forest (Santa Catarina); Coelopterapion Wagner, 1912 to the Pantanal (Mato Grosso), Cerrado and Atlantic Forest (Minas Gerais, São Paulo and Paraná); Mythapion Kissinger, 2005 now includes the Amazon (Amazonas); Stenapion Wagner, 1912 is also extended to include Amazon Forest (Pará), the Pantanal (Mato Grosso and Mato Grosso do Sul), Cerrado and Atlantic Forest (Minas Gerais and Santa Catarina). Apionion samson is reported for the first time from Brazil (Minas Gerais), two species of Coelopterapion, C. cionoides (Wagner 1912a) and C. testaceum (Wagner 1912a) are first records from the state of São Paulo; Trichapion lativentre (Béguin-Billecocq, 1909) is a first record for Paraná; one species of Apion in the broadest sense, A. ensirostre Gerstaecker, 1854, is a first record for the state of Mato Grosso do Sul.

\section{Key to the genera of Brazilian Apioninae (males)}

1. Middle coxae contiguous, not separated by junction of meso- and metaventral processes (Fig.1) ............... Chrysapion Kissinger, 1968

- $\quad$ Middle coxae separated by junction of meso- and metaventral processes (Fig. 2)

2. Elytra expanded and nodose apically; apical prominences in the intervals $2+3$ and 8 interrupted by large deep pit (Fig. 6); if elytra not nodose apically, the apex of intervals $2+3$ and 8 interrupted by punctures; pronotum conical to subconical and without basal flange when in dorsal view Bothryopteron Wagner, 1912a

- $\quad$ Elytra not expanded and nodose apically; apex at intervals 2+3, and 8 not interrupted by large deep pit or punctures; pronotum cylindrical, conical or campaniform to subturbinated or tripartite, with or without basal flange in dorsal view .......................... 3

3. Rostrum straight in side view (Fig. 8), stout and cylindrical pronotum subcylindrical (Fig. 7) to tripartite (Fig. 7) .................

Stenapion Wagner, 1912a 
- $\quad$ Rostrum more or less distinctly curved or tapering towars apex in side view; pronotum conical or campaniform to subturbinate

4. Elytra with fasciate pattern formed by light and/or dark scales (Fig.9); first protarsomere at most isodiametric, second clearly transverse; mesocoxae widely separated (0.25-0.30 times middle coxae diameter) Neapion Alonso-Zarazaga, 1990

Elytra without fasciate pattern; first protarsomere distinctly longer than wide; distance between mesocoxae usually not separated by more than 0.25 mesocoxal diameter (Fig. 2) ..... 6

5. Pronotum subconical, sides in basal two-thirds straight (Fig. 9); body robust; elytra convex to strongly convex, with or without raised areas in intervals (Fig. 10). On Rutaceae

Subgenus Neotropion Alonso-Zarazaga, 1999

- Pronotum subcylindrical, sides rounded; elongated body; elytra never convex to strongly convex, without raised areas in intervals. On Caprifoliaceae

.. Subgenus Neapion Alonso-Zarazaga, 1990

6. Body stout, pyriform and sub-sphaerical elytra in dorsal view; pronotum conical (Fig. 11); elytra strongly convex in lateral view (Fig. 12) Coelopterapion Wagner, 1912a

- Body more elongate; pronotum conical (Fig. 17) to subconical (Fig. 20) or campaniform to subturbinated (Figs. 22, 27); elytra less convex in lateral view (Figs. 17, 18, 21, 23, 28) ............. 7

7. Ventral region of the rostrum with a high median keel, sulci between the median and lateral keel punctate and with semierec pilosity; pronotum campaniform with vestiture perpendicular to the apical margin; elytra with base of the intervals 3 with a dense pubescent spots. On Malvaceae ......................... Alocentron Schilsky 1901 (subgenus Nearctalox Alonso-Zarazaga, 1990)

With another character combination ........................ 8

8. Tegminal plate fused with free ring (Fig. 13-14) Coelocephalapion Wagner, 1914

Tegminal plate articulated with free ring (Fig. 15-16) ........... 9

9. All tibia not mucronate ........ Apionion Kissinger, 1998 (in part)

- At least median tibia mucronate .................................. 10

10. Pronotum conical to subconical, without basal flange (Fig. 20); fore tibia not mucronate .............. Apionion Kissinger, 1998 (in part)

Pronotum otherwise; fore tibia mucronate ................. 11

11. Pronotum with basal flange (Fig. 22) and/or prescutellar fovea; if fore tibia not mucronate, the pronotum is always campaniform (Fig. 22) to subturbinate; first tarsomere of one of the legs modified in the form of plantar spine (Fig. 26) Trichapion Wagner, 1912b

Pronotum without basal flange and prescutellar fovea (Fig. 27); fore tibiae mucronate; tarsomeres not modified in the form of of plantar spine Mythapion Kissinger, 2005a

Checklist of the Apioninae recorded in Brazil

Subfamily Apioninae Schönherr, 1823:1136

Supertribe Apionitae Schönherr, 1823

Tribe Apionini Schönherr, 1823

Subtribe Aspidapiina Alonso-Zarazaga, 1990:34

Genus Alocentron Schilsky, 1901:G (in key)

Type-species: Apion curvirostre Gyllenhal, 1833.
Subgenus Nearctalox Alonso-Zarazaga, 1990:54

Type-species: Apion hibisci Fall, 1918:219.

Synonyms: Apion subgenus Alocentron sensu Kissinger 1968:234, 258 [Alonso-Zarazaga 1990: 54].

Distribution: North, Central and South America (Brazil).

Host plants: Malvaceae.

References: Kissinger 1968:234, 1988:304, 1992:65; Alonso-

Zarazaga 1990:54, 2004:696.

Remarks: examined images of the type species available in The Database of the Zoological Colletions (CVZBASE) of the Museum of Comparative Zoology - Harvard was compared with the studied material.

\section{Alocentron chiriquense (Sharp, 1890)}

Synonyms: Apion chiriquense Sharp, 1890:70; Apion chiriquense sbsp. curvinasus Wagner, 1912b: 100; Apion chiriquense var. curvinasus Wagner, 1912b:118.

Distribution: Mexico, Guatemala, Nicaragua, Panama, Brazil (Rio de Janeiro).

References: Blackwelder 1947:828; Kissinger 1959a:28, 1968:235, 236; O'Brien and Wibmer 1982:21; Wibmer and O'Brien 1986:40.

Subtribe Ixapiina Alonso-Zarazaga, 1990:71

Genus Neapion Alonso-Zarazaga, 1990:75

Type-species: Apion umboniferum Fall, 1898:174.

Synonyms: Apion subgenus Ixias sensu Kissinger 1968:41, 257, not Sainte-Claire Deville, 1924:122 (=Ixapion Roudier et Tempére, 1973:80) [Alonso-Zarazaga 1990: 54]; Xixias Kissinger, 1991:35. Remarks: examined images of the type species available in The Database of the Zoological Colletions (CVZBASE) of the Museum of Comparative Zoology - Harvard University was compared with the studied material.

\section{Subgenus Neotropion Alonso-Zarazaga, 1990:75}

Type-species: Apion xanthoxyli Fall, 1898:176.

Distribution: North, Central and South America (Colombia, Venezuela, Trinidad, Brazil and Paraguay), Antilles.

Host plants: Rutaceae.

References: Kissinger 1959a:24, 31, 1968:42 (as peculiare species group), 1990:37, 1992:65; Alonso-Zarazaga 1990:75, 2004:696; Alonso-Zarazaga and Lyal 1999:58.

Remarks: examined images of the type species available in The Database of the Zoological Colletions (CVZBASE) of the Museum of Comparative Zoology - Harvard University and of the Apion clarki Kissinger, 1990 deposited in the Smithsonian National Museum of Natural History, Washington, EUA (USMN) was compared with the studied material.

Material examined: Neapion (Neotropion) undetermined species. Brazil: Amazonas: Rio Solimões, Lago do José: 1 ind., IIIV-091979, J. Adis, Fumigação (fogging), W. O. Sousa det. (INPA); Acre (New record): Rio Branco: 1 ind., IV-1954, 1 ind., X-1954, M. Alvarenga col., W. O. Sousa det. (MZSP); Pará (New record): Tapará: 1 ind., IX-1922, H. L. Boy, W. O. Sousa det. (MNRJ); 

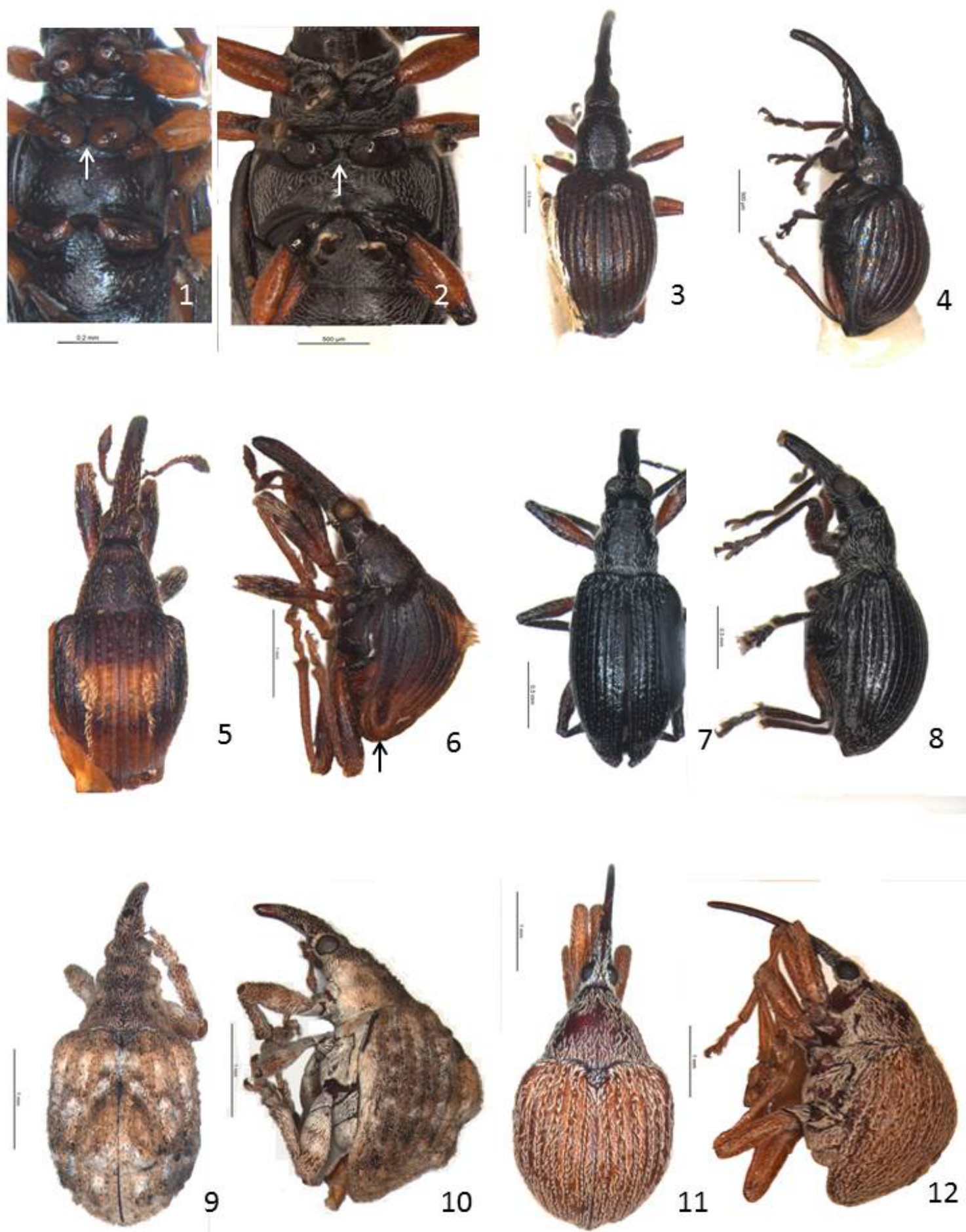

Figures 1-12. Chrysapion sp.: 1, prothorax, meso- and metaventrite ventral view; 3, dorsal view; 4, lateral view. Trichapion sp.: 2 , prothorax, meso- and metaventrite ventral view. Bothryopteron binodosum (Wagner): 5, dorsal view; 6, lateral view. Stenapion sp.: $\mathbf{7}$, dorsal view; 8, lateral view. Neapion (Neotropion) marquesae De Sousa \& Ribeiro-Costa: 9, dorsal view; 10, lateral view. Coelopterapion testaceum (Wagner): 11, dorsal view; 12, lateral view. 
de Sousa, W.O. et al.

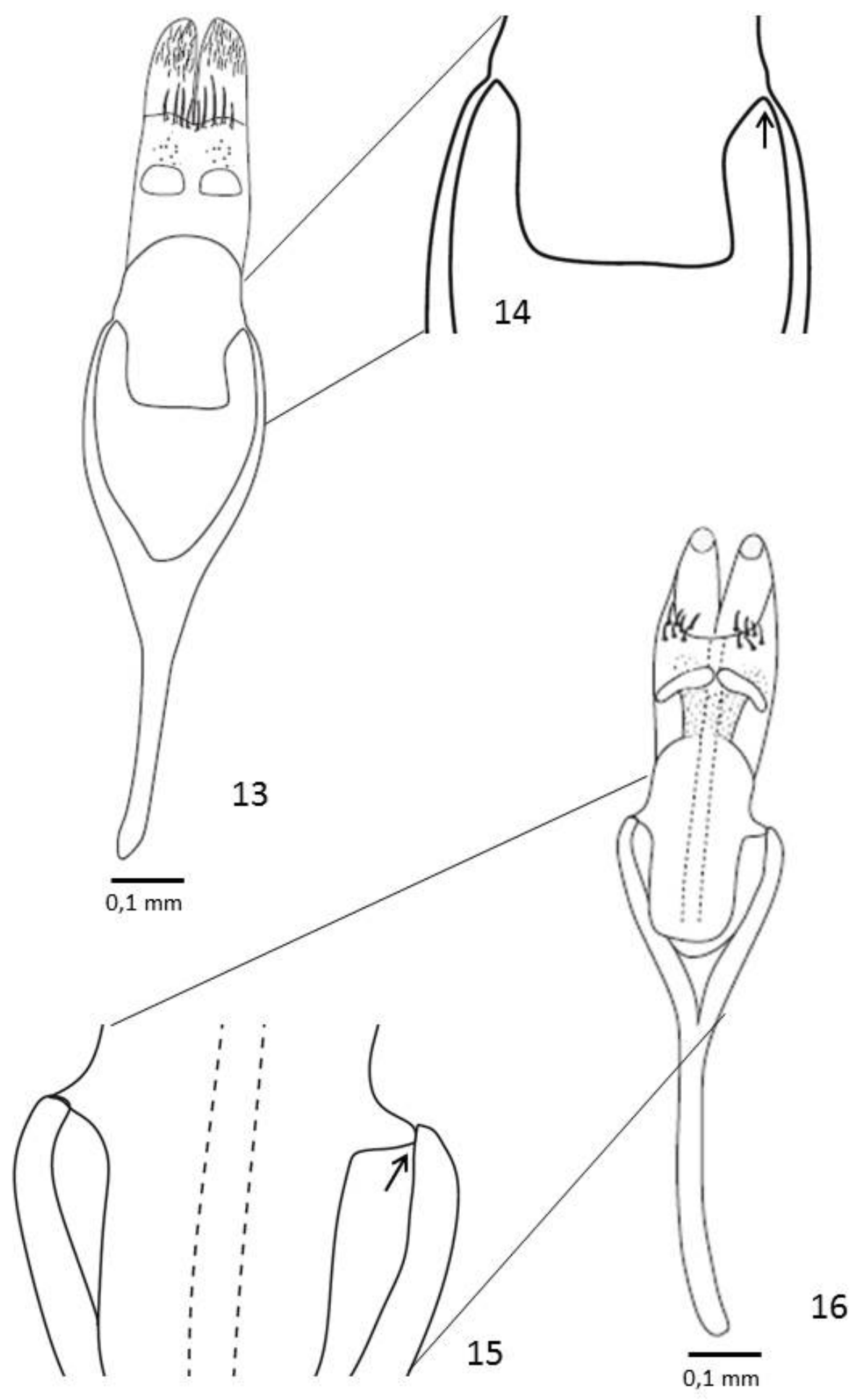

Figures 13-16. Neapion (Neotropion) marquesae De Sousa \& Ribeiro-Costa: 13, Male genitalia: tegmen, dorsal view; 14, prostegium fused with free ring. Trichapion sp. (Béguin-Billecocq): 15, Male genitalia: tegmen, dorsal view; 16, prostegium articulated with free ring. 

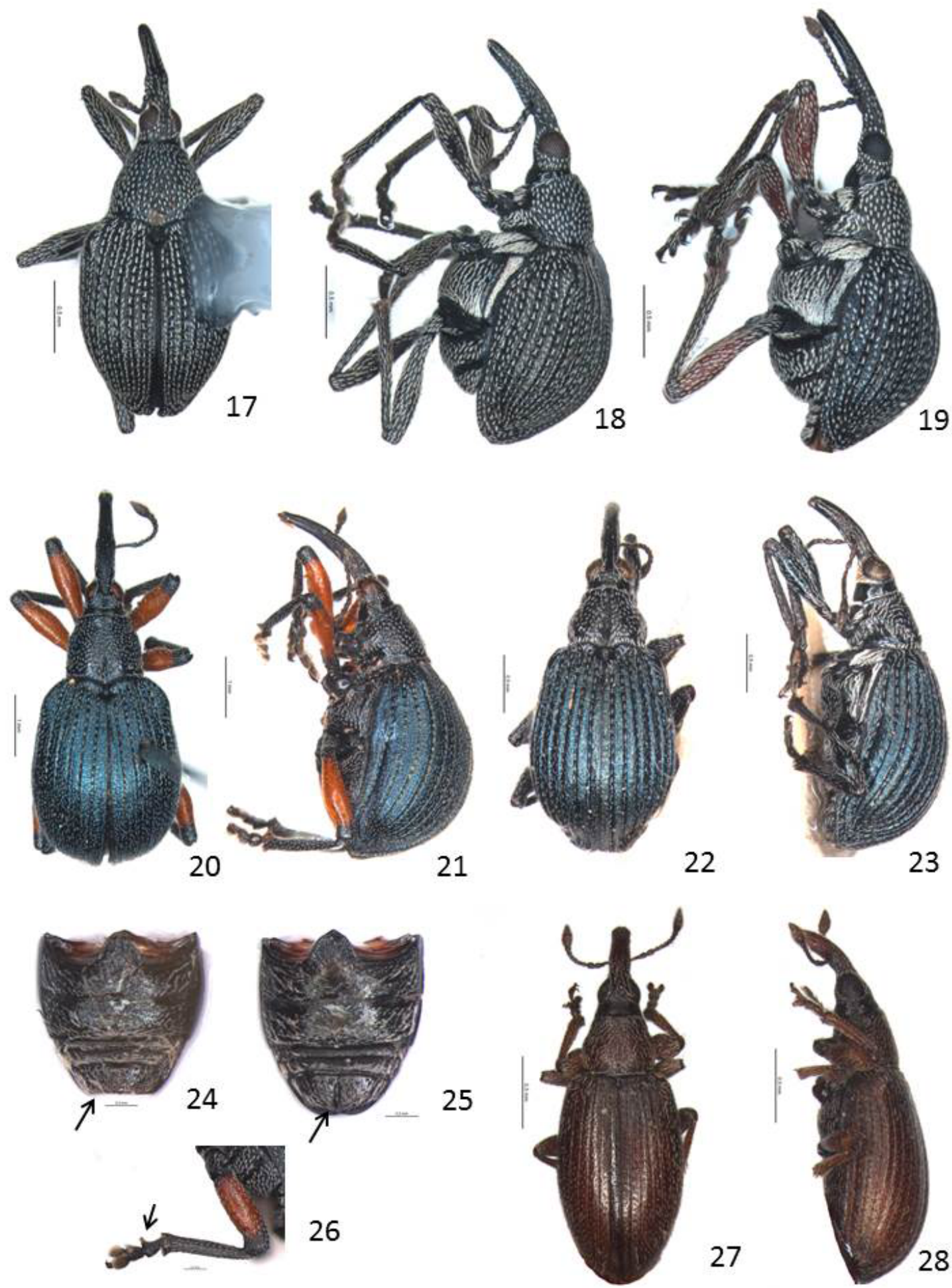

Figures 17-28. Coelocephalapion sp.: 17, male, dorsal view; 18, male, lateral view; 19, female, lateral view. Apionion samson (Sharp): 20, dorsal view; 21, lateral view. Trichapion sp. (Béguin-Billecocq): 22, dorsal view; 23, lateral view; 24, male, abdomen with apical margin of $5^{\text {th }}$ sternite truncate; $\mathbf{2 5}$, female, abdomen with rounded margin and median area of $5^{\text {th }}$ sternite marked by a median sulcus. Trichapion sp.: 26, trochanter, femur, tibia, tarsus 3 and tarsomere 1 modified in the form of plantar spine of male. Mythapion simplex (Béguin-Billecocq): 27, dorsal view; 28, lateral view. 
Mato-Grosso: Barra do Tapirapés: 1 ind., I-30-31-1964, B. Malkin col. (MZSP); São Paulo (New record): Itú: Fazenda Pau D'Alho: 1 ind., I-59, col. U. Martins, W. O. Sousa det. (DZUP); Paraná (New record): Curitiba: 4 ind., XII-1935, 103 (MZSP). Paraguay: Missiones: Villa Florida: 1 male (dissected), IV-17-1988, Colr. C. Aguilar, W. O. Sousa det. (DZUP); San Ignácio, Tahyity: 1 ind., II-20-22-2005, B. Garcete col., W. O. Sousa det. (DZUP).

\section{Neapion (Neotropion) americanum (Wagner, 1908)}

Synonyms: Apion americanum Wagner, 1908:5; Apion conicicolle Sharp, 1890:65 (not Gerstaecker 1854:270) [Wagner, 1908:5]. Distribution: Mexico, Belize, Guatemala, Panama, Costa Rica, Venezuela, Brazil (Goiás: Jataí).

References: Wagner 1910:11, 1912c:117; Blackwelder 1947:828; Kissinger 1963a:156, 1968:41, 42, 1990:37; O'Brien and Wibmer 1982:20; Wibmer and O’Brien 1986:40.

\section{Neapion (Neotropion) diringsi De Sousa \& Ribeiro-Costa, 2018}

Distribution: Brazil (Amazônas: Itacoatiara).

Type material: Brazil, Itacoatiara, Amazonas, Dirings, Januare 1965 (Holotype) (MZUSP). Paratype: 4 females, 1 with genitalia in a separate microvial (MZUSP), glued on paper triangle, same holotype data, 1 female (MZUSP), glued on paper triangle, same holotype data except III/1964.

Material examined: Brazil, Itacoatiara, Amazonas, Dirings, Januare 1965, 1 male without head, glued on paper triangle, with genitalia in a separate microvial (MZUSP).

Neapion (Neotropion) marquesae De Sousa \& Ribeiro-Costa, 2018 Distribution: Brazil (Mato Grosso: Poconé).

Type material: Brasil, Mato Grosso: Poconé, (Canopy), 25/10/2012, Bonatti, J., seca, Cord. 04, Funil 50, Quadrante F2 (Holotype) (DZUP). Paratype: 1 female dissected (DZUP), same holotype information except Cord. 3, Funil 44, 1 female (LETA), same holotype information except 05/09/2013, Funil 36.

\section{Neapion (Neotropion) peculiare (Wagner, 1909)}

Synonyms: Apion peculiare Wagner, 1909:766; Apion gibbosum Sharp, 1891a:82 (not Herbst 1797:103, not Faust 1887) [Wagner, 1909:766].

Distribution: Costa Rica, Panama, Brazil (Goiás: Jataí).

References: Wagner 1912c:117; Blackwelder 1947:830; Kissinger 1963a:158, 1968:41, 44, 1990:38; O’Brien and Wibmer 1982:26; Wibmer and O'Brien 1986:42.

Subtribe Piezotrachelina Voss, 1959a:51

Genus Chrysapion Kissinger, 1968:23

Type-species: Apion auctum Sharp, 1890:78.

Distribution: North, Central, and South America (Colombia, Venezuela and Brazil (New record)), Antilles.

Host plants: Fabaceae.

References: Kissinger 1968:23, 1988:302; O'Brien and Wibmer 1982:19; Wibmer and O'Brien 1986:38; Alonso-Zarazaga 1990:79, 88, 2004:696; Alonso-Zarazaga and Lyal 1998:59.
Remarks: images examined of the material determined by $\mathrm{D}$. G. Kissinger of the species Chrysapion auctum (Sharp, 1890) and Chrysapion tantillum (Sharp, 1890:55) of the Arizona State University Hasbrouck Insect Collectionand (http://symbiota4.acis. ufl.edu/scan/portal/).

Material examined: Chrysapion undetermined species. Brazil (New record): Ceará: Barbalha: 10 ind., V-1969, M. Alvarenga, Coleção M. Alvarenga, W. O. Sousa det. (DZUP), Crato, S. Araripe: 17 ind. V-1969, M. Alvarenga, Coleção M. Alvarenga, W. O. Sousa det. (DZUP); Rio Grande do Norte: Natal: 7 ind., X-1951, M. Alvarenga col., Coleção M. Alvarenga, W. O. Sousa det. (MZSP); Pernambuco: Petrolina: 3 ind., V-1969, M. Alvarenga, Coleção M. Alvarenga, W. O. Sousa det. (DZUP), Caruaru: 2 ind., IV-1972, M. Alvarenga, Coleção M. Alvarenga, W. O. Sousa det. (DZUP).

Subtribe Oxystomatina Alonso-Zarazaga, 1990:110

Genus Coelocephalapion Wagner, 1914:145

Type-species: Apion bryanti Wagner, 1914:147, by subsequent designation of Kissinger 1968:29.

Distribution: North, Central and South America (Colombia, Venezuela, Trinidad, Brazil, Bolivia, Chile and Argentina), Antilles. Introduced in Thailand and Australia.

Host plants: Asteraceae, Euphorbiaceae, Fabaceae.

References: Blackwelder 1947-828, Kissinger 1968:29, 151, 257, 258, 1988:303, 1992:66; O'Brien and Wibmer 1982:20; Wibmer and O'Brien 1986:39; Alonso-Zarazaga 1990:88, 2004:696; Forno et al.1994:147; Alonso-Zarazaga and Lyal 1999:61; Vergara-Pineda et al. 2014.

Material examined: Coelocephalapion undetermined species. Brazil: Pernambuco: Caruaru: 3 ind., IV-1972, M. Alvarenga, Coleção M. Alvarenga, W. O. Sousa det. (DZUP); Mato Grosso (New record): Claudia: 1 male, 3 female, IV-2015, Mod. I, Gap.

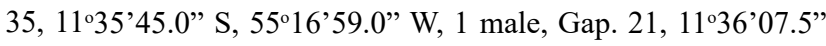

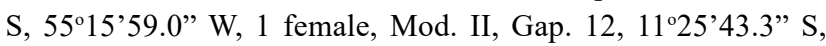
55 18'52.0" W, Puça, Vicente R. E., W. O. Sousa det. (UFMT); Mato Grosso do Sul (New record): Corumbá, Porto Esperança: 1 ind., XII-7-1960, K. Lenko col., Apion nodicorne grp., det Kissinger (MZSP); Rio de Janeiro: Tijuca, Esc. Nac. Agr.: 2 ind. (1 damaged), IV-24- 1935, Charles Hathamay, 11.305, Apion sp. in nodicorne grp. (MZSP), Represa Rio Grande, Guanabara: 1 ind., VII-1972, 3 male, VII-1972, F. M. Oliveira, Coleção M. Alvarenga, W. O. Sousa det. (DZUP); Paraná: Guarapuava, Est. Águas Claras: 1 ind., I-02-1987, Lev. Ent. PROFAUPAR LAMPADA, Ponta Grossa: 1 ind. VII-20-87, Prof. Mal., W. O. Sousa det. (DZUP).

\section{Coelocephalapion atrirostre (Fabricius, 1802)}

Synonyms: Attelabus atrirostre Fabricius, 1802:424;.Apion luteirostre Gerstaecker, 1854:254 [Alonso-Zarazaga, 2014:75]; Apion acarinum Sharp 1890:64 [Wagner 1912a:30]; Apion argentinum Béguin-Billecocq 1909:464 [Wagner 1912a:30]; Apion cydoniae Kissinger 1957:40, error [not Bondar, 1950:454]. Distribution: Mexico, Guatemala, Panama, Colombia, Venezuela, Brazil (Rio de Janeiro, Santa Catarina), Surinam, Bolivia, Argentina. 
References: Wagner 1910:8; Blackwelder 1947:829; Kissinger 1959a:30, 1968::196, 210; O'Brien and Wibmer 1982:24; Wibmer and O'Brien 1986:42.

Type material: 1 ind., Apion argentinum L. B. B, Rep. Argentina, Prov. Buenos Aires, VII-09-1905, C Bruch (Holotype) (MACN). Material examined: Brazil: Rio de Janeiro: Represa Rio Grande, Guanabara: 1 ind., VII-1962, F. M. Oliveira, Coleção M. Alvarenga, W. O. Sousa det. (DZUP).

\section{Coelocephalapion brachyrrhinum (Wagner, 1914)}

Synonyms: Apion brachyrrhinum Wagner, 1914:150.

Distribution: Brazil (Rio de Janeiro).

References: Blackwelder 1947:828; Kissinger 1968:168; Wibmer and O'Brien 1986:40.

Remarks: lost type material (Kissinger 1968:168).

\section{Coelocephalapion coelocephalum (Wagner, 1914)}

Synonyms: Apion coelocephalum Wagner, 1914:146.

Distribution: Brazil (Rio de Janeiro).

References: Blackwelder 1947:828; Kissinger 1968168; Wibmer and O'Brien 1986:40.

Remarks: lost type material (Kissinger 1968:168).

Coelocephalapion dissimilipes (Sharp, 1890:49)

Synonyms: Apion dissimilipes Sharp, 1890:49.

Distribution: Mexico, Guatemala, Panama, Brazil (Pernambuco e Bahia).

References: Wagner 1912a:32; Blackwelder 1947:829; Kissinger 1963b:122, 125, 1968:171, 172; O'Brien and Wibmer 1982:22; Wibmer and O’Brien 1986:41.

\section{Coelocephalapion longipenne (Wagner, 1912a:13)}

Synonyms: Apion longipenne Wagner, 1912a:13; Apion cydoniae Bondar, 1950:454 [Kissinger 1968:195]; Apion luteirostre Kissinger 1957:40 [not Gerstaecker, 1854:254].

Distribution: Mexico, Brazil (Pernambuco e Bahia).

Host plants: Croton sp. (Euphorbiaceae).

References: Blackwelder 1947:829; Kissinger 1959a:30, 1968:195, 210; D'Araujo e Silva 1968:461; O'Brien and Wibmer 1982:24; Vaurie 1953:14; Wibmer and O'Brien 1986:42.

Type material: 6 ind., Apion cydoniae Bond., Estado da Bahia, Brazil, G. Bondar Leg. (Cotype) (MNRJ).

\section{Coelocephalapion rugifrons (Boheman, 1839)}

Synonyms: Apion rugifrons Boheman, 1839:373.

Distribution: Brazil.

References: Wagner 1910:55; Blackwelder 1947:830; Kissinger 1968:168, Wibmer and O'Brien 1986:43.

\section{Coelocephalapion spretissimum (Sharp, 1890)}

Synonyms: Apion spretissimum Sharp, 1890:48.

Distribution: Mexico, Belize, Costa Rica, EI Salvador, Guatemala, Nicaragua, Panama, Brazil (Rio de Janeiro).

References: Blackwelder 1947:830; Kissinger 1963b:122, 126, 1968:174, 209; O'Brien and Wibmer 1982:27; Wibmer and O'Brien 1986:43.
Subtribe Trichapiina Alonso-Zarazaga, 1990:116

Genus Apionion Kissinger, 1998:93

Type-species: Apion crassum Fall, 1898:165.

Distribution: North, Central and South America (Colombia, Trinidad, Brazil, Peru and Bolivia).

Host plants: Asteraceae, Euphorbiaceae, Fabaceae.

References: Kissinger 1968:152 (as annulatum species group), 1998:93; Alonso-Zarazaga and Lyal 1999:60; Whitehead 1977:165 (as Coelocephalapion annulatum species group).

Remarks: examined images of the type of the Apion neolentum Kissinger, 1968 available in The Database of the Zoological Colletions (CVZBASE) of the Museum of Comparative Zoology - Harvard University was compared with the studied material.

Material examined: Apionion undetermined species. Brazil: Manaus (New record): Reserva Duke, $26 \mathrm{Km}$ NE Manaus: 1 male, Plot C Malaise 3, IV-95, Barbosa, M. G. V., W. O. Sousa det. (INPA), Rio Solimões, Lago do José, Prox. Manaus: 2 male, VIII-09-1979, fumigação (fogging), J. Adis e outros, W. O. Sousa det. (INPA); Mato Grosso (New record): Nossa Senhora do Livramento, Baia dos Coqueiros: 2 female, IX-25-1988, Marinez I. Marques, W. O. Sousa det. (UFMT); Rio de Janeiro (New record): Rio de Janeiro, (Corcovado) GB: 2 male, IX-1958, 1 male, X-1958, 1 male, 1 female, XI-1958, 1 male., X-1959, 4 male (1 dissected), 2 female, VIII-1960, Corcovado, D. Federal: 1 male., 1 female, VIII-1958, M. Alvarenga, Ex-coleção M. Alvarenga, W. O. Sousa det. (MZSP).

Apionion annulatum (Gerstaecker, 1854)

Synonyms: Apion annulatum Gerstaecker, 1854:256.

Distribution: Brazil.

References: Wagner 1910:11, Blackwelder 1947:828, Kissinger 1968:152, 1998:93; Wibmer and O’Brien 1986:40.

\section{Apionion faldermanni (Gyllenhal, 1839)}

Synonyms: Apion faldermanni Gyllenhal, 1839:370.

Distribution: Brazil.

References: Wagner 1910:27; Blackwelder 1947:829; Kissinger 1968:152; Wibmer and O'Brien 1986:41.

\section{Apionion samson (Sharp, 1891)}

Synonyms: Apion samson Sharp, 1891:84.

Distribution: Belize, Panamá, Bolivia, Brazil.

References: Kissinger 1968:49; O'Brien and Wibmer 1982:21; Wibmer and O'Brien 1986:43.

Material examined: Brazil (New record): Minas Gerais: Rio Verde: 440m, 1 male, Cu, 447, 11.60, W. O. Sousa det. (DZUP).

\section{Genus Trichapion Wagner, 1912b:116}

Type-species: Apion aurichalceum Wagner, 1912b:103, by subsequent designation Kissinger 1959b:248.

Distribution: North, Central and South America (Colombia, Venezuela, Brazil, Peru, Bolivia, Chile, Paraguay, Argentina and Uruguay). Introduced in South Africa.

Host plants: Asteraceae, Fabaceae.

References: Blackwelder 1947:828, Kissinger 1959a:24, 32, 1959b:247, 1968:49; 1989a:271; O'Brien and Wibmer 1982:20; Wibmer and O'Brien 1986:39; Alonso-Zarazaga 1990:116, 
2004:696; Alonso-Zarazaga and Lyal 1999:59; Alonso-Zarazaga and Wanat 2014:399, 405.

Material examined: Trichapion undetermined species. Brazil: Amazonas: Parque Nacional do Jaú, Rio Carabinani, mg. Dir 1'59'S/61'32'W: 1 male, IV-06-07-1994, Motta, C, et al. Col., Luz mista mercúrio Luz negra BL e BLB Lençol, W. O. Sousa det. (INPA), Manaus, Reserva Duke, $26 \mathrm{Km}$ NE Manaus: 1 male, Plot C Malaise 4, 1 female, Plot C Malaise 1, 1 female, Plot C Malaise 5, 1 female, 372, 0031505, Plot C Malaise 4, VI-95, 1 female, 372, 0031506, 1 female, 372, 0031507, Plot C Malaise 4, V-95, Barbosa, M. G. V., W. O. Sousa det. (INPA).

\section{Trichapion acanthopus (Wagner, 1912)}

Synonyms: Apion acanthopus Wagner, 1912c:110.

Distribution: Brazil (Amazonas), Bolivia.

References: Blackwelder 1947:828; Wibmer and O’Brien 1986:39.

\section{Trichapion fusconitidum (Wagner, 1912)}

Synonyms: Apion fusconitidum Wagner, 1912a:23.

Distribution: Brazil (Rio de Janeiro: Petrópolis).

References: Blackwelder 1947:829; Kissinger 1959b:250, 332, 338;

Wibmer and O'Brien 1986:41.

Trichapion lativentre (Béguin-Billecocq, 1909)

Synonyms: Apion lativentre Béguin-Billecocq, 1909:451.

Distribution: Brazil (Rio de Janeiro), Paraguay, Argentina, Uruguay. Introduced in South Africa.

References: Wagner 1912a:32; Blackwelder 1947:829; Kissinger 1959b:250, 369, 374; Wibmer and O'Brien 1986:41; AlonsoZarazaga 1990:148; Bachmann and Lanteri 2013:210; AlonsoZarazaga and Wanat 2014:339, 405.

Type material: 1 ind., Apion lativentre tipo L. B. B, Rep. Argentina, Prov. Buenos Aires, III-1897, C Bruch (Syntype) (MACN).

Material examined: Brazil: Paraná (New record): Curitiba: 6 male, IV-12-1980, 13 male (1 dissected), 19 female (1 dissected), XII-5-1980, Rosado Neto col., W. O. Sousa det. (DZUP).

\section{Trichapion longicorne (Wagner, 1912)}

Synonyms: Apion longicorne Wagner, 1912c:107.

Distribution: Brazil (Amazonas), Bolivia.

References: Blackwelder 1947:829; Wibmer and O’Brien 1986:42.

\section{Trichapion spinitarse (Wagner, 1912)}

Synonyms: Apion spinitarse Wagner, 1912a:18.

Distribution: Nicaragua, Brazil (Rio de Janeiro).

References: Blackwelder 1947:830; Kissinger 1959b:-250, 254, 321, 1968:68, 70, 74; Wibmer and O'Brien 1986:43.

Trichapion suffuscum (Wagner, 1912)

Synonyms: Apion suffuscum Wagner, 1912b:113.

Distribution: Brazil (Rio de Janeiro).

References: Blackwelder 1947:830; Wibmer and O'Brien 1986:43.
Incertae sedis genera

Genus Bothryopteron Wagner, 1912a:8

Type-species: Apion grallarium Sharp, 1891a:83, by subsequent designation Kissinger 1968:29 (examined images of the material available in Kissinger (1968)).

Distribution: North, Central and South America (Brazil and Bolivia).

Host plants: unknown.

References: References: Kissinger 1959a:26, 28, 1968:32, 243; O'Brien and Wibmer 1982:20; Wibmer and O'Brien 1986:39; Alonso-Zarazaga 2004:696; Alonso-Zarazaga and Lyal 1998:61.

Material examined: Bothryopteron undetermined species. Brazil: Maranhão (New record): Igarape Gurupi - Uma aldeia Araçú: 1 ind., V-1968, B. Malkin col., W. O. Sousa det. (MZSP); Mato-Grosso (New record): Utiariti $(325 \mathrm{~m})$, Rio Papagaio: 1 ind., CII-VIII-961, K. Lenko col., W. O. Sousa det. (MZSP), Chapada dos Guimarães, Chapada Aventura: 1 ind., VIII-232016, UmbrellaEnt, A. O. Lira leg., W. O. Sousa det. (LETA), Rio Caraguata: 3 ind., III-1953, F. Plaumann, Coleção Campos Seabra, W. O. Sousa det. (MNRJ); Tocantins (New record): Dianápolis: 1 ind., I-16-22-1962, J. Bechyné col., W. O. Sousa det. (MZSP); Goiás (New record): Pirineus: 1 ind., II-2-1962, J. Bechyné col., W. O. Sousa det. (MZSP); Rio de Janeiro: Rio de Janeiro, Recreio dos Bandeirantes, Baia da Guanabara: 2 ind., dz 48/60, V-8-60, J. C. M. Carvalho, W. O. Sousa det. (MNRJ); Santa Catarina (New record): Nova Teutônia: 1 ind., $27^{\circ} 11^{\prime}$ 'B. 52 23' 2 ' XI-1953, 1 ind. I-1954, Fritz Plaumann col., W. O. Sousa det., (MZSP), 1 ind., XII1976, 300-500 m, Fritz Plaumann col., W. O. Sousa det. (DZUP).

\section{Bothryopteron balzani (Wagner, 1912)}

Synonyms: Apion balzani Wagner, 1912a:9.

Distribution: Brazil (Rio de Janeiro), Bolivia.

References: Blackwelder 1947:828, Wibmer and O'Brien 1986:40.

\section{Bothryopteron binodosum (Wagner, 1912)}

Synonyms: Apion binodosum Wagner, 1912a:10.

Distribution: Brazil (Rio de Janeiro, Bahia).

References: Wagner 1912c:117; Wibmer and O'Brien 1986:40. Material examined: Brazil: Rio de Janeiro: Represa Rio Grande, Guanabara: 1 ind., X-1960, Coleção Campos Seabra, F. M. Oliveira, W. O. Sousa det. (MNRJ), 1 ind., IX-1961, Coleção M. Alvarenga, F. M. Oliveira, W. O. Sousa det. (DZUP).

\section{Bothryopteron praestabile (Wagner, 1914)}

Synonyms: Apion praestabile Wagner, 1914:159.

Distribution: Brazil.

References: Blackwelder 1947:830; Wibmer and O'Brien 1986:43.

\section{Bothryopteron steinbachi (Wagner, 1912)}

Synonyms: Apion steinbachi Wagner, 1912a:11.

Distribution: Brazil (Amazonas), Bolivia.

References: Blackwelder 1947:830; Wibmer and O'Brien 1986:43. 


\section{Genus Coelopterapion Wagner, 1912a}

Type-species: Apion testaceum Wagner, 1912a:3, by subsequent designation Kissinger 1968:29.

Distribution: South America (Brazil and Paraguay).

Host plants: Euphorbiaceae.

References: References: Lucas 1920:196; Kissinger 1968:33; O'Brien and Wibmer 1982:20; Wibmer and O'Brien 1986:39; Alonso-Zarazaga 2004:696; Alonso-Zarazaga and Lyal 1998:61. Material examined: Coelopterapion undetermined species. Brazil: Mato Grosso (New record): Rio Caraguata: 1 ind., VI-1953, $218^{\circ}$ 48'B. 52 27'L, 400 m, Fritz Plaumann, W. O. Sousa det. (MZSP); Minas Gerais (New record): Pouso Alegre: 3 ind., XII-1965, F. S. Pereira col. Sousa, W. O., det. (MZSP); Espirito Santo: Sta. Tereza: 1 ind., XII-7-64, C. Elias leg. W. O. Sousa det. (DZUP); São Paulo (New record): Botucatu: 3 ind. III-1967, Dirings, W. O. Sousa det. (MZSP), Barueri: 1 ind., X-15-1954, 1 ind., I-22-1961, 1 ind., IV-9-1964, K. Lenko col., W. O. Sousa det. (MZSP), São Paulo, Cantareira: 1 ind. II-17-1960, J. Halik, 3626, W. O. Sousa det. (MZSP), Ferraz de Vasconcelos: 1 ind., XI-19-1971, J. J. Ferraciolli, 3117, Coleção Ferraciolli, W. O. Sousa det. (MZSP), Salesópolis, E. B. da Boracéia: 1 ind., X-2007, Col. Uehara M., W. O. Sousa det. (UFMT), Ilha dos Búzios: 1 ind., X-16-XI-04-963, Exp. Dep. Zool., W. O. Sousa det. (MZSP); Rio de Janeiro: Itatiaia, Est. Biológica: 1 ind., XI-29-32, 700m, W. Zikán, W. O. Sousa det. (MNRJ); Rio de Janeiro: Realengo, Guanabara: 1 ind., X-9-1949, M. Alvarenga leg., Ex-coleção M. Alvarenga, W. O. Sousa det. (MZSP), Corcovado, Rio, Guanabara: 1 male (dissected), IX-1958, Alvarenga e Seabra, Ex-coleção M. Alvarenga, W. O. Sousa det. (MZSP), (Corcovado) GB: 1 ind., X-1958, M. Alvarenga, Ex-coleção M. Alvarenga, W. O. Sousa det. (MZSP), Inst. Biolog. Entomologia Agrícola: 1 ind., III-1930, coll Dario Mendes, W. O. Sousa det. (MNRJ); Paraná (New record): Londrina: 1 ind., II-14-75, Pe. Moure, Rosado leg, 1 ind., XII-6-8-75, Pe. Moure leg., W. O. Sousa det. (DZUP), Jundiai do Sul, Fazenda Monte Verde: 1 ind., X-5-1986, Lev. Ent. PROFAUPAR, Lampada, W. O. Sousa det. (DZUP).

\section{Coelopterapion cionoides (Wagner, 1912)}

Synonyms: Apion cionoides Wagner, 1912a:5.

Distribution: Brazil (Rio de Janeiro, Pernambuco).

Host plants: Croton floribundus (Euphorbiaceae).

References: Blackwelder 1947:828; D’Araujo e Silva 1968:461; Kissinger 1968:33; Wibmer and O'Brien 1986:40.

Material examined: Brazil: São Paulo (New record): Barueri: 1 ind., X-7-1954, 97, leg K. Lenko, det. DKiss 1957 (MZSP).

\section{Coelopterapion fuscipenne (Wagner, 1912)}

Synonyms: Apion fuscipenne Wagner, 1912a:7.

Distribution: Brazil (Bahia).

References: Blackwelder 1947:829; Wibmer and O’Brien 1986:41.

\section{Coelopterapion globulum (Wagner, 1912)}

Synonyms: Apion globulum Wagner, 1912a:6.

Distribution: Brazil (Rio de Janeiro, Bahia).

References: Blackwelder 1947:829; Wibmer and O'Brien 1986:41.
Coelopterapion subulirostre (Gyllenhal, 1833)

Synonyms: Apion subulirostre Gyllenhal, 1833:266; Apion daimio Sharp, 1891b:296 [Kissinger 1989b:24]; Apion fryi Wagner, 1912a:4 [Kissinger 1989b:24].

Distribution: Brazil (Pernambuco, Rio de Janeiro).

References: Wagner 1910-61; Blackwelder 1947-829, 830; Wibmer and O'Brien 1986-43.

\section{Coelopterapion testaceum (Wagner, 1912)}

Synonyms: Apion testaceum Wagner, 1912a:3.

Distribution: Brazil (Rio de Janeiro).

References: Blackwelder 1947:830; Kissinger 1968:29, 33; Wibmer and O'Brien 1986:43.

Material examined: Brazil: São Paulo (New record): Barueri: 1 ind., X-14-1955, K. Lenko col., det Kissinger (MZSP).

\section{Genus Mythapion Kissinger, 2005a:85}

Type-species: Mythapion trifolianum Kissinger, 2005a:86.

Distribution: South America (Brazil, Chile, Argentina and Uruguay).

Host plants: unknown.

References: Kissinger 2005a:85.

Material examined: Mythapion undetermined species. Brazil: Amazonas (New record): Manaus: ZF-2. Km-19: 1 male, VIII18-1979, J. Adis e outros, fumigação (fogging), W. O. Sousa det. (INPA).

\section{Mythapion simplex (Béguin-Billecocq, 1909)}

Synonyms: Apion simplex Béguin-Billecocq, 1909:459.

Distribution: Brazil (Bahia, Rio de Janeiro), Argentina, Uruguay. References: Wagner 1912a:31; Blackwelder 1947:830; Kissinger 2005a:71, 86, 88; Wibmer and O'Brien 1986:43; Bachmann and Lanteri 2013:210.

Material examined: Argentina: Buenos Aires: 1 ind., I, 2-y2, 373, Coleção F. Justus Jor. (DZUP), 2 ind., II-1942, Dirings (MZSP).

\section{Genus Stenapion Wagner, 1912a:20}

Type-species: Apion constricticolle Sharp, 1890:59, by subsequent designation Kissinger 1968:29 (examined images of the material available in Kissinger (1968)).

Distribution: North, Central and South America (Trinidad, Brazil, Peru, Bolivia, Paraguay and Argentina).

Associated plants: Polygonaceae.

References: Wagner 1915:35; Kissinger 1959a:23, 32, 1968:29, 39, 127; O'Brien and Wibmer 1982:20; Wibmer and O'Brien 1986:39; Alonso-Zarazaga 2004-696; Alonso-Zarazaga and Lyal 1999:61. Type material: 1 ind., Apion salpingoides $=$ mediocre L. B. B, Rep. Argentina, Prov. Buenos Aires, II-2-1905, C Bruch (Syntype), 1 ind., Apion contrarium L. B. B, Rep. Argentina, Prov. Buenos Aires, XI-27-1904, C Bruch (Syntype) (MACN).

Material examined: Stenapion undetermined species. Brazil: Pará (New record): Camargo-Itaituba, Rio Tapajós: 1 ind., XI-1963, Dirings, W. O. Sousa det. (MZSP); Mato-Grosso (New record): Poconé: Pirizal: 4 ind., Armadilha luminosa, Cambará-Borda, 
I-15-2000, W. O. Sousa col., W. O. Sousa det. (LETA), Baia do Coqueiro: 1 ind. X-2000 P.4, Gaiola de ADIS, W. O. Sousa col., W. O. Sousa det. (LETA), Barão de Melgaço: 1 ind., Baia9/P03 Litorânea, IX-02-09, Brizzola and Sousa col., W. O. Sousa det. (LETA); Mato Grosso do Sul (New record): Costa Rica: 1 ind., XII-18-87, F. Sta Cruz. Exp. IQ. MZ DZIQUSP, W. O. Sousa det. (MZSP); Minas Gerais (New record): Santa Barbara, Serra da Caraça: 1 ind. XI-23-25-960, Araujo e Martins (MZSP); Rio de Janeiro: Represa Rio Grande, Guanabara: 2 ind., VII-1972, F. M. Oliveira, W. O. Sousa det. (DZUP); Santa Catarina (New record): Nova Teutônia: 2 ind., $27^{\circ} 11^{\prime}$ B. 52 $2^{\circ}$ 23'L, XI-1953, Fritz Plaumann col., W. O. Sousa, det. (MZSP), 1 ind., XII-1976, 300-500 m, Fritz Plaumann col., W. O. Sousa, det. (DZUP). Paraguay: Puerto La Esperanza: La Victoria, Alto-Paraguay: 1 ind. XI-30-XII-3-2006, B. Garcete col., $79 \mathrm{msnm} 22^{\circ} 01$ '30,7'S - 50 01'34,9'W, Malaise trap 1 in dense Schinopsis-Ruprechtia forest, W. O. Sousa det. (DZUP); Asuncion: 2 ind., I-30-22-1985, col. R. T. Bonace, Bahia de Asuncion vegetacion flotante, W. O. Sousa det. (DZUP).

\section{Stenapion brevinasus (Wagner, 1912)}

Synonyms: Apion brevinasus Wagner, 1912a:20.

Distribution: Brazil (Pará: Monte Alegre, S. Antonio-Brasilia), Paraguay, Argentina, Uruguay.

References: Wagner 1915:41, 52; Blackwelder 1947:828; Wibmer and O'Brien 1986:40.

\section{Stenapion carinifrons (Wagner, 1914)}

Synonyms: Apion carinifrons Wagner, 1914:152.

Distribution: Brazil.

References: Wagner 1915:39, 59; Blackwelder 1947:828; Wibmer and O'Brien 1986:40.

\section{Stenapion divergens (Wagner, 1912)}

Synonyms: Apion divergens Wagner, 1912a:21.

Distribution: Brazil (Rio de Janeiro).

References: Blackwelder 1947:829; Wagner 1915:41, 46; Wibmer and O'Brien 1986:41.

\section{Stenapion heydeni (Wencker 1863)}

Synonyms: Apion heydeni Wencker 1863:181.

Distribution: Brazil (Monte Alegre), Bolivia, Paraguay, Argentina.

References: Wagner 1910:33, 1915:40, 52; Blackwelder 1947:829; Wibmer and O'Brien 1986:41.

\section{Stenapion yatahyanum (Wagner, 1912)}

Synonyms: Apion yatahyanum Wagner, 1912c:116.

Distribution: Brazil (Goiás: Jatai).

References: Wagner 1915:38, 59; Blackwelder 1947:829; Wibmer and O'Brien 1986:44.

Incertae sedis species ("Apion" in the broadest sense)

Apion alocorhinum Wagner, 1914:141

Distribution: Brazil (Goiás: Jatai).

References: Blackwelder 1947:828; Wibmer and O’Brien 1986:40.
Apion ampullula Gerstaecker 1854:255

Synonyms: Apion macromerum Kirsch, 1874:419 [Wagner 1912a:31].

Distribution: Peru, Brazil.

References: Wagner 1910:11; Blackwelder 1947:828; Wibmer and O’Brien 1986:40.

\section{Apion angulatum Gerstaecker 1854:245}

Distribution: Brazil (Goiás: Jatai).

References: Wagner 1910:11; Blackwelder 1947:828; Wibmer and O’Brien 1986:40.

Apion angustifrons Wagner, 1914:162

Distribution: Brazil (Rio de Janeiro: Teresópolis).

References: Blackwelder 1947:828; Wibmer and O’Brien 1986:40.

Apion areolatum Kirsch, 1874:423

Distribution: Peru, Brazil (Amazonas, Rio de Janeiro).

References: Wagner 1912a:32, Blackwelder 1947:828; Wibmer and O'Brien 1986:40.

Remarks: examined images of the type of the A. areolatum deposited in the Staatliches Museum für Tierkunde, Dresden.

Apion atronitidum Wenker, 1863:178

Distribution: Brazil (São Paulo).

References: Wagner 1910:14; Blackwelder 1947:828; Wibmer and O’Brien 1986:40.

Apion bicoloratum Wagner, 1914:138

Distribution: Brazil (Pernambuco).

References: Blackwelder 1947:828; Wibmer and O’Brien 1986:40.

Apion brachypterum Wagner, 1912b:130

Distribution: Brazil (Amazonas, Rio de Janeiro).

References: Blackwelder 1947:828; Wibmer and O'Brien 1986:40.

Apion brasilianum Wagner, 1912a:14

Distribution: Brazil (Pernambuco, Rio de Janeiro).

References: Blackwelder 1947:828; Wibmer and O'Brien 1986:40.

Apion brevicorne Gerstaecker 1854:249

Distribution: Brazil.

References: Wagner 1910:15; Blackwelder 1947:828; Wibmer and O'Brien 1986:40.

Material examined: Brazil: Rio de Janeiro, Tijuca, Esc. Nac. Agr., Brasil Central: 1 ind., IX-1945, C. de Araujo, 11.308, det. Kissinger (MZSP).

Apion brevipes Wagner, 1912a:25

Distribution: Brazil (Pernambuco).

References: Blackwelder 1947:828; Wibmer and O'Brien 1986:40.

Apion coeruleosquamosum Wagner, 1912b:131

Distribution: Peru, Bolivia, Brazil (Amazonas).

References: Blackwelder 1947:828; Wibmer and O'Brien 1986:40. 
Apion convergens Wagner, 1912a:28

Distribution: Brazil (Pernambuco, Rio de Janeiro).

References: Blackwelder 1947:828; Wibmer and O'Brien 1986:41.

Apion convexirostre Wagner, 1912b:135

Distribution: Brazil (Amazonas).

References: Blackwelder 1947:828; Wibmer and O'Brien 1986:41.

Apion dentinasus Wagner, 1912b:133

Distribution: Brazil (Rio de Janeiro).

References: Blackwelder 1947:829; Wibmer and O'Brien 1986:41.

Apion desbordesi Béguin-Billecocq, 1911:131

Distribution: Brazil (Ceará), Argentina.

References: Wagner 1912a:31; Blackwelder 1947:829; Wibmer and O'Brien 1986:41; Bachmann and Lanteri 2013:209.

Material examined: Syntype: 1 female, Apion desbordesi L. B. B, Rep. Argentina, Prov. Cordoba, 190, C Bruch (MACN).

\section{Apion ensirostre Gerstaecker, 1854:251}

Synonyms: Apion columbianum Faust, 1894:319 [Wagner

1912a:31].

Distribution: Colombia, Bolivia, Brazil.

References: Wagner 1910:19, 1912c:118; Blackwelder 1947:829; Wibmer and O'Brien 1986:41.

Material examined: Brazil: Mato Grosso do Sul (New record): Corumbá, Porto Esperança: 1 ind., XII-7-1960, K. Lenkko col., 129, det. Kissinger (MZSP).

Apion exophthalmum Wencker, 1863:179

Distribution: Brazil.

References: Wagner 1910:26; Blackwelder 1947:829; Wibmer and O’Brien 1986:41.

Apion fulvicorne Wagner, 1912a:29

Distribution: Brazil (Rio de Janeiro).

References: Blackwelder 1947:829; Wibmer and O’Brien 1986:41.

Apion gilvirostre Wagner, 1912a:26

Distribution: Brazil (Amazonas).

References: Blackwelder 1947:829; Wibmer and O'Brien 1986:41.

Apion inaequale Gyllenhal, 1833:256

Distribution: Brazil.

References: Wagner 1910:34; Blackwelder 1947:829; Wibmer and O'Brien 1986:41.

Apion informe Béguin-Billecocq, 1911:131

Distribution: Bolivia, Brazil (Rio de Janeiro).

References: Wagner 1912a:32, 1912c:117; Blackwelder 1947:829; Wibmer and O'Brien 1986:41.
Apion ingratum Béguin-Billecocq, 1909:461

Distribution: Brazil (Amazonas), Argentina.

References: Wagner 1912a:32; Blackwelder 1947:829; Wibmer and O'Brien 1986:41; Bachmann and Lanteri 2013:210.

Type material: 1 ind., Apion ingratum L. B. B, Rep. Argentina, Prov. Buenos Aires, 190, C. Bruch (Syntype) (MACN).

Apion latifrons Gerstaecker, 1854:246

Distribution: Brazil.

References: Wagner 1910:37; Blackwelder 1947:829; Wibmer and O’Brien 1986:41.

Apion lividipes Wencker, 1863:180

Distribution: Brazil.

References: Blackwelder 1947:829; Wibmer and O'Brien 1986:42.

Apion longitarse Wagner, 1914:141

Distribution: Brazil.

References: Blackwelder 1947:829; Wibmer and O'Brien 1986:42.

Apion longulum Gerstaecker, 1854:239

Distribution: Brazil.

References: Wagner 1910:39; Blackwelder 1947:829; Wibmer and O’Brien 1986:42.

Apion luridipes Wagner, 1912a:24

Distribution: Brazil.

References: Blackwelder 1947:829; Wibmer and O'Brien 1986:42.

Apion luteipes Kirsch, 1874:419

Distribution: Peru, Brazil.

References: Wagner 1910:39; Blackwelder 1947:829; Wibmer and O’Brien 1986:42.

Remarks: examined images of the type of the A. luteipes deposited in the Staatliches Museum für Tierkunde, Dresden.

Apion macrostylum Wagner, 1914:144

Distribution: Brazil (Rio de Janeiro: Rio de Janeiro; Minas Gerais: Matusinho, Caraça).

References: Blackwelder 1947:829; Wibmer and O’Brien 1986:42.

Apion nigroaeneum Gerstaecker, 1854:255

Distribution: Brazil.

References: Wagner 1910:43; Blackwelder 1947:829; Wibmer and O’Brien 1986:42.

Apion nigrosuturatum Béguin-Billecocq, 1909:459

Distribution: Brazil (São Paulo), Argentina.

References: Wagner 1912:32; Blackwelder 1947:829; Wibmer and O’Brien 1986:42. 
Apion novaeteutoniae Voss, 1959b:315 [in footnote]

Distribution: Brazil (Nova Teotonia).

References: Weidner 1976:147; Wibmer and O'Brien 1986:42.

Remarks: examined images of the holotype, 1 female of the $A$. novaeteutoniae deposited in the Zoological Museum, Hamburg.

\section{Apion opacum Kirsch, 1874:420}

Distribution: Peru, Brazil.

References: Wagner 1910:45; Blackwelder 1947:830; Wibmer and O’Brien 1986:42.

Remarks: examined images of the type of the $A$. opacum deposited in the Staatliches Museum für Tierkunde, Dresden.

\section{Apion parviceps Wagner 1914:160}

Distribution: Brazil.

References: Blackwelder 1947:830; Wibmer and O'Brien 1986:42.

\section{Apion parvulum Gerstaecker, 1854:248}

Synonyms: Apion motabile Faust, 1894:317 [Wagner, 1910:47].

Distribution: West Indies, Colombia, Venezuela, Brazil.

References: Wagner 1910:47, 1912a:31; Blackwelder 1947:830; Wibmer and O'Brien 1986:42.

\section{Apion periscelis Gyllenhal, 1839:391}

Synonyms: Apion strangulatum Kirsch, 1874:413 [Wagner, 1910:47].

Distribution: Peru, Bolivia, Brazil.

References: Wagner 1910:47, 1912a:31; Blackwelder 1947:830; Wibmer and O'Brien 1986:42.

Material examined: Brazil: Minas Gerais: Pouso Alegre: 1 ind., IX-962, F. S. Pereira col., 139, det. Kissinger (MZSP).

\section{Apion phaseoli Voss, 1958:921}

Distribution: Brazil (Bahia).

References: Weidner 1976:147; Wibmer and O'Brien 1986:43. Remarks: examined images of the paratype of the $A$. phaseoli deposited in the Zoological Museum, Hamburg.

\section{Apion pseudampullula Voss, 1940:12}

Synonyms: Apion pseudampulla [Bondar 1950:452, error].

Distribution: Brazil.

Host plants: Leguminosae.

References: D'Araujo e Silva 1968:461; Wibmer and O'Brien 1986:43.

Material examined: 2093: 2 ind., Voss (MNRJ).

Apion pulverulentum Wencker, 1863:177

Distribution: Brazil.

References: Wagner 1910:51; Blackwelder 1947:830; Wibmer and O’Brien 1986:43.

\section{Apion pygmaeum Wagner, 1912a:29}

Distribution: Brazil (Rio de Janeiro).

References: Blackwelder 1947:830; Wibmer and O'Brien 1986:43.
Apion retusipenne Wagner, 1912a:28

Distribution: Brazil.

References: Blackwelder 1947:830; Wibmer and O'Brien 1986:43.

Apion rubrirostre Wagner, 1912a:13

Distribution: Brazil (Rio de Janeiro).

References: Blackwelder 1947:830; Wibmer and O'Brien 1986:43.

Apion rubronigrum Wagner, 1912b:132

Distribution: Brazil (Bahia, Rio de Janeiro).

References: Blackwelder 1947:830; Wibmer and O'Brien 1986:43.

Apion sesbaniae Voss, 1958:919

Distribution: Brazil (Rio Grande do Sul: Porto Alegre).

References: Weidner 1976:147; Wibmer and O'Brien 1986:43.

Remarks. Examined images of the paratype, 1 female of the $A$. sesbaniae deposited in the Zoological Museum - Hamburg.

Apion subnudum Wagner, 1912a:21

Distribution: Brazil (Goiás: Jataí), Bolivia.

References: Blackwelder 1947:830; Wibmer and O'Brien 1986:43.

\section{Apion tropidorrhynchum Wagner, 1914:143}

Distribution: Brazil.

References: Blackwelder 1947:830; Wibmer and O'Brien 1986:44.

Apion tucumanense Béguin-Billecocq, 1909:458

Distribution: Brazil (Rio de Janeiro), Bolivia, Argentina.

References: Wagner 1912c:118; Blackwelder 1947:830; Wibmer and O'Brien 1986:44; Bachmann and Lanteri 2013:211.

Type material: 1 female, Apion tucumanense L. B. B, Rep. Argentina, Prov. Tucuman, I-20-1904, C. Bruch (Syntype) (MACN).

Apion undulipenne Wagner, 1912a:11

Distribution: Brazil (Bahia).

Host plants: Cassia apoucouita (Fabaceae).

References: Voss 1940:12; Blackwelder 1947:830; D’Araujo e Silva 1968:461; Wibmer and O'Brien 1986:44.

Material examined: Brazil: Bahia: 5 ind., 1977, G. Bondar leg., Voss deter., 5 ind., 1977 (MNRJ).

Apion wingelmulleri Wagner, 1912a:14

Distribution: Brazil (Rio de Janeiro).

References: Blackwelder 1947:831; Wibmer and O'Brien 1986:44.

Apion xanthopus Gyllenhal, 1839:375

Synonyms: Apion sulcipenne Gyllenhal, 1839:401 [Wagner, 1912a:31]).

Distribution: Brazil.

References: Wagner 1910:61,67, 1912a:31; Blackwelder 1947:831; Wibmer and O'Brien 1986:44.

Apion xanthorhynchum Wagner, 1912a:27

Distribution: Brazil.

References: Blackwelder 1947:831; Wibmer and O'Brien 1986:44. 


\section{Apion zikani Heller, 1922:52}

Distribution: Brazil (Minas Gerais: Passa Quatro).

Host plants: Dalbergia glaucescens (Mart. ex. Benth), D. foliolosa (Benth) (Fabaceae).

References: Blackwelder 1947:831; Costa-Lima 1956:126; D'Araujo e Silva 1968:461; Wibmer and O'Brien 1986:44.

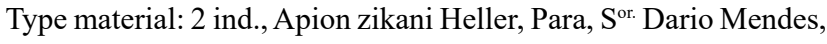
Passa Quatro Minas Zikan (Cotype) (MNRJ).

\section{Discussion}

We recognize 10 genera of Brazilian Apioninae, one of which is a first record for Brazil (Chrysapion) and which appears to be restricted to northeastern Brazil. Three genera are widely distributed in the New World: Alocentron, Coelocephalapion and Trichapion. The latter two genera are found throughout Brazil. Five genera (Apionion, Bothryopteron, Coelopterapion, Neapion and Stenapion) have more restricted distributions in South America (Alonso-Zarazaga and Lyal 1999) but also are found throughout Brazil. Coelopterapion is the only genus with most species subendemic to Brazil (Kissinger 1968) and whose distributions are restricted to the Cerrrado and Atlantic Forest. The recently described Mythapion is monospecific and found in Argentina, Brazil and Uruguay (Wibmer and O’Brien 1986; Kissinger 2005a), from the Amazon to the Pampas of South America.

Only 86 species of Apioninae from Brazil were described from 1833 to 1959 , mostly by Hans Wagner, with the recent addition of two new species (De Sousa and Ribeiro-Costa 2018). Yet, Alonso-Zarazaga (pers. comm. 2017) reported that Don Whitehead estimated that the number of Brazilian Apioninae species is closer to 1,500, most of which are endemic. Alonso-Zarazaga and Wanat (2014) suggested 800 species for the Neotropical region, which is likely to be a large underestimation. More than 50 years have passed since the last Brazilian species were described. Thus, our descriptions and keys provide a new landmark for the taxonomy of the Apioninae in South America. Brazil is among the most biodiverse countries with many hotspots (Aleixo et al. 2010; Marques and Lamas 2006; Myers et al. 2000) and so the Apioninae is a very promising group and much more promising comparing parazitoid hymenopterans wasp or dipterans in South America.

We identified ca 230 especimens to genus or species. Taxonomic impediments, such as brief, incomplete, original descriptions, often without illustrations that would facilitate species recognition, prevent identification at the species level for most. Additionally, many small specimens apparently comprise a large number of morphotypes, but each morphotype included only a few specimens without marked sexual dimorphism. Thus, many specimens remain to be identified.

Upon examination of the Apioninae in Brazilian collections we found a large quantity of unidentified material, many specimens of which are only labelled Apion sp., while others were mixed Apioninae. We found no specimen that was identified with any generic name other than Apion, and there were no subgenera. Hence the challenge of this study, in which we provide a list for the Brazilian taxa and place them in genera and subgenera. All identification was through comparison with relevant diagnostic characters of the original descriptions, with the addition of currently available types or images of type material or with material previously identified by specialists.
External morphology and genitalia of males, both of which are fundamental for identification of taxa in the Apioninae, are described in Alonso-Zarazaga $(1989,1990,2004)$ and Alonso-Zarazaga \& Wanat (2014). Also, efficient use of the key requires the separation of morphotypes and identification of the sex of each morphotype. Species identification based only on females is essentially impossible and so males are required. For that end, an adequate collection with many specimens that can be dissected to identify sex is required (Alonso-Zarazaga 2004). If dissection is not possible, secondary sexual dimorphism must be identified and which can be observed in the rostrum (shorter in males, Fig. 18, wider and generally more coarsely sculptured and pubescent than in females, Fig. 19; Anderson \& Kissinger 2002). Some males have mucro in at least one pair of tibiae, or the first tarsomere of one of the legs is modified as plantar spines (Fig. 26). The apical margin of ventrite 5 is truncated with a part of the pygidium being visible externally, or the pygidium is formed by the 8 th tergite ( 7 th in females, Fig. 24). In females, the legs have no special characters and ventrite 5 (Fig. 25) has a rounded margin (Alonso-Zarazaga 1990). Rarely, ventrite 5 of females (in some species) may have a median glabrous area or a median sulcus (Alonso-Zarazaga and Wanat 2014), such as in Trichapion sp. (Fig. 25).

Hundreds of specimens in South American collections are still waiting to be studied, many of them likely to be new taxa. Thus, these specimens may be among the 10 genera comprising the key, or they may be unknown genera or even be among the non-monophyletic hyperdiverse genera (Trichapion and Coelocephalapion). Thus, they demonstrate the potential of the group for future systematic research at all taxonomic levels. Additionally, studies must be carried out to recover monophyly of the genera and tribes and to clarify relationships of incertae sedis, all based on morphology, molecular, biogeography and host plant associations.

\section{Acknowledgements}

The first author thanks to the Fundação de Amparo à Pesquisa do Estado de Mato Grosso (FAPEMAT - No. 10/2016) for the granting postdoctoral fellowship outside the State of Mato Grosso and Programa de Pós-Graduação em Entomologia da Universidade Federal do Paraná of the support. The second author thanks the Conselho Nacional de Desenvolvimento Científico e Tecnológico (CNPq) for providing scholarship. We are greatful to curators accompanied for loan the material examined: Lúcia Massuti de Almeida (DZUP), Fernando Zagury Vaz de Melo (UFMT), Márcio Luiz de Oliveira (INPA), Marinêz Isaac Marques (LETA); Marcela Laura Monné Freire (MNRJ), Sônia A. Casari (MZSP). To Martin Husemann (Zoological Museum - Hamburg), Olaf Jaeger (Staatliches Museum für Tierkunde - Dresden) and Lourdes Chamorro and Mr. Ashton Smith (Smithsonian Institution) by the images of the types. To Arturo Roig Alsina for the visit to the Museu Argentino de Ciencias Naturales "Bernardino Rivadavia" for the study of Apioninae types.

\section{Author Contributions}

Wesley Oliveira de Sousa: substantial contribution in the concept and design of the study; data collection; analysis and interpretation; manuscript preparation and critical revision, adding intelectual content. 
Cibele Stramare Ribeiro-Costa: contribution in the concept and design of the study; manuscript preparation and critical revision, adding intelectual content.

Germano Henrique Rosado-Neto: contribution in the concept and design of the study and critical revision, adding intelectual content.

\section{Conflicts of interest}

The author(s) declare(s) that they have no conflict of interest related to the publication of this manuscript.

\section{References}

ALEIXO, A., ALBERNAZ, A.L., GRELLE, C.E.V., VALE, A.M. \& RANGEL, T.F. 2010. Mudanças climáticas e a biodiversidade dos biomas brasileiros: Passado, Presente e Futuro. Nat. Conserv. 8 (2): 194-196. https://doi. org/10.4322/natcon.00802016

ALONSO-ZARAZAGA, M.A. 1989. Revision of the supraspecific taxa in the Palaearctic Apionidae Schoenherr, 1823. 1. Introduction and subfamily Nanophyinae Seidlitz, 1891 (Coleoptera, Curculionoidea). Fragm. Entomol. 21 (2): 205-262.

ALONSO-ZARAZAGA, M.A. 1990. Revision of the supraspecific taxa in the Palaearctic Apionidae Schoenherr, 1823 (Coleoptera, Curculionoidea). 2. Subfamily Apioninae Schoenherr, 1823: Introduction, keys and descriptions. Graellsia 46: 19-156

ALONSO-ZARAZAGA, M.A. 2004. Apionidae (Coleoptera). In Biodiversidad, taxonomía y biogeografía de artrópodos de México: hacia una síntesis de su conocimiento. Vol. IV (J. L. Bousquets, J.J. Morrone, O. Yañez \& I. Vargas, eds). Facultad de Ciencias, Universidad Nacional Autónoma de México y Comisión Nacional para el Conocimiento y Uso de la Biodiversidad, Mexico, D. F., p. 691-699.

ALONSO-ZARAZAGA, M.A. 2014. On the identity of some weevil species described by Johann Christian Fabricius (1745-1808) in the Museum of Zoology of Copenhagen (Coleoptera, Cucujoidea, Curculionoidea, Tenebrionoidea). ZooKeys 451: 61-91.

ALONSO-ZARAZAGA, M.A. \& LYAL, C.H.C. 1999. A World catalogue of Families and Genera of Curculionoidea (Insecta: Coleoptera) (Excepting Scolytidae and Platypodidae). Barcelona, Spain, Entomopraxis, S. C. P.

ALONSO-ZARAZAGA, M.A. \& WANAT, M. 2014. Apioninae Schoenherr, 1823. In Handbook of Zoology. Arthropoda: Insecta: Coleoptera, beetles. Vol. 3. Morphology and systematics (Phytophaga) (R.A.B. Leschen, R.G. Beutel RG, eds). de Gruyter, Berlin, p. 395-415.

ANDERSON, R.S. \& KISSINGER, D.G. 2002. 129. Brentidae Billberg 1820. In American Beetles Volume 2. Polyphaga: Scarabaeoidea through Curculionoidea (R.H Arnett Jr., M.C Thomas, P.E. Skelley, J.H. Frank, eds). CRC Press, Boca Raton, p. 711-719.

BACHMANN, A.O. \& LANTERI, A.A. 2013. Catalog of the types osf Curculionoidea (Insecta, Coleoptera) deposited at the Museo Argentino de Ciencias Naturales "Bernardino Rivadavia", Buenos Aires. Rev. Mus. Argent. Cienc. Nat. 15 (2): 209-280.

BADENES-PEREZ, F.R. \& JHONSON, M.T. 2007. Ecology and impacto of Allorhogas sp. (Hymenoptera: Braconidae) and Apion sp. (Coleoptera: Curculionoidea) on fruits of Miconia calvescens DC (Melastomataceae) in Brazil. Biol. control 43: 317-322. https://doi.org/10.1016/j. biocontrol.2007.08.007

BÉGUIN-BILLECOCQ, L. 1909. Apions nouveaux de la République Argentine [Col. Curculionidae]. Ann. Soc. Entomol. Fr. 78: 449-464.

BÉGUIN-BILLECOCQ, L. 1911. Contribution à la faune des Coléoptères de l'Amérique du Sud. Diagnoses d'espèces nouvelles d'Apionidae [Col. Curculionidae]. Bull. Soc. Entomol. Fr.: 131-133.

BLACKWELDER, R.E. 1947. Checklist of the Coleopterous insects of Mexico, Central America, the West Indies, and South America. Part 5. Bull. U. S. Natl Mus. 185.
BOHEMAN, C.H. 1839. In SCHOENHERR, C.J. (op. cit).

BONDAR, G. 1950. Notas entomológicas da Baía. XXII. Rev. Entomol. 21(3): 449-480.

BOUCHARD, P., BOUSQUET, Y., DAVIES, A.E., ALONSO-ZARAZAGA, M.A., LAWRENCE, J.F., LYAL, C.H.C., NEWTON, A.F., REID, C.A.M., SCHMITT, M., SLIPNSKI, A.S., SMITH, A.B.T. 2011. Famlily-group names in Coleoptera (Insecta). Zookeys 88: 1-972. https://doi.org/10.3897/ zookeys. 88.807

COSTA-LIMA, A. 1956. Insetos do Brasil. Coleópteros. $10^{\circ}$ Tomo. Coleópteros, $4^{\mathrm{a}}$. e última parte. Escola Nacional de Agronomia, Rio de Janeiro. Série Didática $\mathrm{n}^{\circ} 12$.

D'ARAÚJO E SILVA, A.G., GONÇALVES, C.R., GALVÃO, D.M., GONÇALVES, A.J.L., GOMES, J., SILVA, M.N. \& SIMONI, L. 1968. Quarto Catálogo dos Insetos que Vivem nas Plantas do Brasil, seus Parasitos e Predadores - ParteII - $1^{\circ}$ Tomo, Fundação IBGE, Rio de Janeiro.

DE SOUSA, W.O. \& RIBEIRO-COSTA, C.S. 2018. Two new Brazilian species of Neapion Alonso-Zarazaga, 1990 subgenus Neotropion Alonso-Zarazaga, 1990 with a key to South American species (Brentidae: Apioninae). Zootaxa 4402 (3): 551-562.

FALL, H.C. 1898. Revision of the species of Apion of America north of Mexico. Trans. Am. Entomol. Soc. 25: 105-184.

FALL, H.C. 1918. New North American species of Apion. J. N. Y. Entomol. Soc. 26 (3-4): 218-223.

FAUST, J. 1887. Curculioniden aus dem Amur-Gebiet. Deutsc. Entomol. Zeitschr. 31 (1): 161-180.

FAUST, J. 1894. Reise von E. Simon in Venezuela. Curculionidae. Stett. Entomol. Ztg. 54 (10-12): 313-367, 1893 (1894).

FORNO, W., HEARD, T.A. \& DAY, M.D. 1994. Host specificity and aspects of the biology of Coelocephalapion aculeatum (Coleoptera: Apionidae), a potential biological control agent of Mimosa pigra (Mimosaceae). Environ. Entomol. 23 (1): 147-153. https://doi.org/10.1093/ee/23.1.147

GERSTAECKER, [C.E.] A. 1854. Beschreibung neuer Arten der Gattung Apion Herbst. Stettiner Entomol. Ztg. 15(8): 234-261, (9), 267-280.

GYLLENHAL, L. 1833. In SCHOENHERR, C.J. (op. cit).

GYLLENHAL, L. 1839. In SCHOENHERR, C.J. (op. cit)

HELLER, K.M. 1922. Springende Blütenkelche, verursacht durch ein neues Apion (Col.). Entomol. Mitt. 11 (2): 52-54.

HERBST, J.F.W. 1797. Natursystem aller bekannten in= und ausländischen Insekten, als eine Fortsetzung der von Büffonschen Naturgeschichte. Pauli, Berlin, Der Käfer, VII, Theil.

JONES, R.W., NIÑO-MALDONADO, S. \& LUNA-COZAR, J. 2012. Diversity and biogeographic affinities of Apionidae (Coleoptera: Curculionoidea) along an altitudinal gradient in El Cielo Biosphere Reserve of northeastern Mexico. Rev. Mex. Biod. 83: 100-109.

KIRSCH, T.F.W. 1874. Beiträge zur Kenntniss der Peruanischen Käferfauna auf Dr. Abendroth's Sammlungen basirt. Berl. Entomol. Zeitschr. 18: 385-432.

KISSINGER, D.G. 1957. Taxonomic notes on North American Apion. Proc. Entomol. Soc. Wash. 59 (1): 40.

KISSINGER, D.G. 1959a. The Species groups of Apion occurring in North and Central America (Curculionidae). Coleopt. Bull. 13 (1): 21-32.

KISSINGER, D.G. 1959b. A revision of the Apion subgenus Trichapion Wagner in the New World (Coleoptera Curculionidae). Proc. U. S. Natl. Mus. 110: 247-389.

KISSINGER, D.G. 1963a. Studies on North American Apion: the Apion peculiare group. Proc. Entomol. Soc. Wash. 65 (2): 153-163.

KISSINGER, D.G. 1963b. North American Apion: the Apion spretissimum group (Coleoptera: Curculionidae). Coleopt. Bull. 17 (4): 121-127.

KISSINGER, D.G. 1968. Curculionidae subfamily Apioninae of North and Central America with reviews of the world genera of Apioninae and world subgenera of Apion Herbst (Coleoptera). Taxonomic Publications. South Lancaster, Massachusetts. 
KISSINGER, D.G. 1988. New host and distribution records for Apionidae from North and Central America (Coleoptera). Coleopt. Bull. 42 (3): 302-304.

KISSINGER, D.G. 1989a. Apionidae from North and Central America. Part 1. Notes on the classification of the Apion subgenus Trichapion Wagner with description of new species from the United States of America (Coleoptera). Insecta Mundi 3 (4): 271-227.

KISSINGER, D.G. 1989b. Synonymy for Apion daimio Sharp, a Brazilian species erroneously attributed to Japan (Coleoptera: Apionidae). Coleopt. Bull. 43 (1): 24.

KISSINGER, D.G. 1990. Apionidae from North and Central America. Part 2. Description of a new subgenus and two new species of Apion from Mexico. (Coleoptera). Insecta Mundi 4 (1-4): 33-40.

KISSINGER, D.G. 1991. Apionidae from North and Central America. Part 3. A new genus in the tribe Aplemonini Kissinger from Mexico (Coleoptera). Insecta Mundi 5 (1): 19-23.

KISSINGER, D.G. 1992. Apionidae from North and Central America. Part 4. Generic classification and introduction to the genus Coelocephalapion Wagner, with new species from Mexico and Venezuela (Coleoptera). Insecta Mundi 6 (2): 65-77.

KISSINGER, D.G. 1998. Apionidae from North and Central America. Part 5. Description of the genus Apionion and 4 new species (Coleoptera). Insecta Mundi 12 (1-2): 93-102.

KISSINGER, D.G. 1999a. Apionidae from North and Central America. Part 6. Description of new species of Apionion Kissinger, Coelocephalapion Wagner and Trichapion Wagner (Coleoptera). Insecta Mundi 13 (1-2): 21-37.

KISSINGER, D.G. 1999b. Description of a new genus, Sayapion, from North and Central America (Coleoptera: Apionidae). Insecta Mundi 13 (1-2): 72.

KISSINGER, D.G. 2002. A New Genus of Apionidae from Chile and Argentina. Coleopt. Bull. 56 (2): 315-316.https://dx.doi.org/10.1649/0010065X(2002)056[0315:SN]2.0.CO;2

KISSINGER, D.G. 2003. A new species of Coelocephalapion Wagner (Apionidae) from Venezuela with host Hyptis suaveolens (L.) Poit. (Lamiaceae). Coleopt. Bull. 57 (1): 99-104. https://dx.doi.org/10.1649/0010065X(2003)057[0099:ANSOCW]2.0.CO;2

KISSINGER, D.G. 2005a. Review of Apioninae of Chile (Coleoptera: Curculionoidea: Apionidae). Coleopt. Bull. 59 (1): 71-90.https://dx.doi. org/10.1649/0010-065X(2005)059[0071:ROAOCC]2.0.CO;2

KISSINGER, D.G. 2005b. A New Species of Coelocephalapion Wagner (Coleoptera: Curculionoidea: Apionidae: Apioninae) from Argentina and Chile Associated with the Genus Prosopis L. (Fabaceae). Coleopt. Bull. 59 (4): 493-500.

KUSCHEL, G. 1995. A phylogenetic classification of Curculionoidea to families and subfamilies. Mem. Entomol. Soc. Wash. 14: 5-33.

LIMA, E.C., PAIVA R., NOGUEIRA, R.C., SOARES, F.P., EMRICH, E.B. \& SILVA, A.A.N. 2008. Callus induction in leaf segments of Croton urucurana Baill. Cienc. Agrotecnol. 32 (1): 17-22.

LUCAS, R. 1920. Catalogus alphabeticus generum et subgenerum coleopterorum orbis terrarum totius (famil., trib., subtrib., sect., incl,). pars 1. Arch. naturgesch. 84, A (1), I-XXI+1-128, (2), 129-288, (3): 289-448.

MAIA, C.M. 2012. Coleopterous galls from the Neotropical Region. Pap. Avulsos Dep. Zool. 52 (15): 175-184.

MARQUES, A.C. \& LAMAS, C.J.E. 2006. Taxonomia zoológica no Brasil: estado da arte, expectativas e sugestões de ações futuras. Pap. Avulsos Dep. Zool. 46 (13): 139-174.

MARVALDI, A.E., SEQUEIRA, A.S., O'BRIEN, C.W. \& FARRELL, B.D. 2002. Molecular and morphological phylogenetics of weevils (Coleoptera: Curculionoidea): do niche shifts accompany diversification? Syst. Biol. 51 (5): 761-785. https://doi.org/10.1080/10635150290102465

MYERS, N., MITTERMEIER, R.A., MITTERMEIER, C.G., FONSECA, G.A.B. \& KENT, J. 2000. Biodiversity hotspots for conservation priorities. Nature 403: 853-858.

OBERPRIELER, R.G., MARVALDI, A.E. \& ANDERSON, R.S. 2007. Weevils, weevils, weevils everywhere. Zootaxa 1668: 481-520.
O'BRIEN, C.W. \& WIBMER, G.J. 1982. Annotated checklist of the weevils (Curculionidae sensu lato) of North America, Central America, and the West Indies (Coleoptera: Curculionoidea). Mem. Am. Entomol. Inst. 34.

PTASZYNSKA, A.A., LETOWSKI, J., GNAT, S. \& MATEK, W. 2012. Application of COI sequence in studies of phylogenetic relationship among 40 Apionidae species. J. Insect. Sci. 12: 1-14.

RADENES-PEREZ, F.R. \& JHONSON, M.T. 2007. Ecology and impacto of Allorhogas sp. (Hymenoptera: Braconidae) and Apion sp. (Coleoptera: Curculionoidea) on fruits of Miconia calvescens DC (Melastomataceae) in Brazil. Biol. Control 43: 317-322.

SAINTE-CLAIRE DEVILLE, J. 1924. Faune des Coléoptères du Bassin de la Seine par Louis Bedel. Supplément aux Rhynchophora Rédigé d'après les notes de L. Bedel. Vol. VI bis, Paris, Société Entomologique de France, Publications Hors Série, pp: 1-80,1923, pp: 81-159, 1924.

SCHILSKY, J. 1901. Die Käfer Europa's nach der Natur beschrieben von Dr. H. C. Küster und Dr. G. Kraatz. Nürnberg. 38 Heft: -VI + A-K + 100 nrs.

SCHÖNHERR, C.J. 1823. Curculionides [Tabula synoptica familiae Curculionidum]. Isis von Oken, 10: 1132-1146.

SCHÖNHERR, C.J. 1833. Genera et species curculionidum cum synonymia hujus familiae: species novae aut hactenus minus cognitae, descriptionibus a Dom. Leonardo Gyllenhall, C. H Boheman, et entomologis aliis illustratae. Lipsiae, Fleischer, Roret, Paris, Vol. 1, pt. 1, I-XV, 1-381.

SCHOENHERR, C.J. 1839. Genera et species curculionidum cum synonymia hujus familiae: species novae aut hactenus minus cognitae, descriptionibus a Dom. Leonardo Gyllenhall, C. H Boheman, et entomologis aliis illustratae. Lipsiae, Fleischer, Roret, Paris, Vol. 5 (1): 1-456.

SHARP, D. 1889-1911. Biologia Centrali-Americana. Insecta. Coleoptera. Rhynchophora. Curculionidae. Attelabinae, Pterocolinae, Allocoryninae, Apioninae, Thecesterninae, Otiorhynchinae [part, “Apterae"], vol. 4, pt.3, pp. 41-80 (1890), 81-168 (1891a), illus.

SHARP, D. 1891b. The Rhynchophorous Coleoptera of Japan. Part ii: Apionidae and Anthribidae. Trans. Entomol. Soc. Lond. 1891 (2): 293-328.

VERGARA-PINEDA, S., JONES, R.W., MALDA-BARRERA, G., CALTZONTZIN-FERNANDÉZ, K., OBREGÓN-ZÚÑIGA, A. \& CAMBRÓN-SANDOVAL, V.H. 2014. Coelocephalapion subornatum (Fall) (Coleoptera: Brentidae: Apioninae) forms galls in stems of Acacia farnesiana (L.) Willd. In Central Mexico. Southwest. Entomol. 40 (1): 223-226. https://doi.org/10.3958/059.040.0121

VAURIE, P. 1953. The Gregorio Bondar types of South American weevils (Coleoptera, Curculionidae). Am. Mus. Novit. 1615: 1-39.

VOSS, E. 1940. Über neue und wenig bekannte Rüssler-Arten aus der neotropischen Region, Vorwiegend aus Brasilien. (Coleoptera: Curculionidae). Arb. Morphol. Taxon. Entomol. Berlin-Dahlem 7 (1): 1-16.

VOSS, E. 1958. Einige unbeschriebene Curculioniden, vorwiegend aus dem Museum G. Frey. (Col. Curc.). Entomol. Arb. Mus. Frey, 9 (3): 918-933.

VOSS, E. 1959a. Ein Beitrag zur Kenntnis der Apioniden-Fauna Zentralafricas (Col. Curc.). Ann. Mus. R. Congo Belge (Sér. 8. Sci. Zool.), 76: 7-119.

VOSS, E. 1959b. Bemerkungen zu einigen Apionen, vorwiegend typischen Exemplaren aus dem Musée Royal du Congo Belge. Rev. Zool. Bot. Afr. 60 (3-4): 313-325.

WAGNER, H. 1908. Die südafrikanischen Apionden des British Museum, vorzugsweise von Herrn G.-A.-K. Marshall im Mashonalande und in Natal gesammelt. Mém. Soc. Entomol. Belg. 16: 1-62.

WAGNER, H. 1909. Zur Synonymie der Gattung Apion Hbst. (Col.). Deutsch. Entomol. Zeitsch.: 766-767.

WAGNER, H. 1910. Curculionidae: Apioninae. In Coleopterorum Catalogus auspiciis et auxilio W. Junk (SCHENKLING, S. ed.). Junk, Berlin.

WAGNER, H. 1912a Beitrag zur Kenntnis der Apion-Fauna Central- und Süd-Amerikas. Beschreibungen neuer Arten, nebst synonymischen Bemerkungen. I. Teil. Mém. Soc. Entomol. Belg. 19: 1-32.

WAGNER, H. 1912b. Beitrag zur Kenntnis der Apion-Fauna Central- und Süd-Amerikas. Beschreibungen neuer Arten. II. Theil. Arch. Naturgesch. 78A (2): 99-136. 
de Sousa, W.O. et al.

WAGNER, H. 1912c. Neue Apionen des Nord- und Südamerikanischen Faunengebietes. Novit. Zool. 19: 97-118.

WAGNER, H. 1914. Beitrag zur Kenntnis der Apion-Fauna Zentral- und SüAmerikas. Beschreibungen neuer Arten. III. Teil. Arch. Naturgesch. 79, A (9), 1913(1914): 137-164.

WAGNER, H. 1915. Revision der bisher Bekannten Arten des Apion-Subgenus Stenapion Wagn. (Col., Curcul.). Entomol. Mitt. 4 (1-3): 35-62.

WANAT, M. 2001. Genera of Australo-Pacific Rhadinocybinae and Myrmacicelinae with biogeography of the Apionidae (Coleoptera: Curculionoidea) and phylogeny of the Brentidae (s. lato). Mantis, Olsztyn.

WEIDNER, H. 1976. Die Entomologischen Sammlungen des Zoologischen Instituts und des Zoologischen Museums der Universität Hamburg. IX. Teil. Insecta VI. Mitt. Hamb. Zool. Mus. Inst. 73: 87-264.
WENCKER, [E]. 1863. Description de plusieurs espèces nouvelles exotiques du genre Apion Herbst. Ann. Soc. Entomol. Fr. 3: 177-182.

WHITEHEAD, D.R. 1977. New Apion (Coelocephalapion) Species (Curculionidae: Apioninae) from fruits of Pterocarpus (Leguminosae: Faboidea) in Central America. Coleopt. Bull. 31(2): 165-172.

WIBMER, G.J. \& O'BRIEN, C.W. 1986. Annotated checklist of the weevils (Curculionidae sensu lato) of South America (Coleoptera: Curculionoidea). Mem. Am. Entomol. Inst. 39.

WINTER, S., FRIEDMAN, A.L., ASTRIN, J.J., GOTTSBERGER, B. \& LETSCH, H. 2017. Timing and host plant associations in the evolution of the weevil tribe Apionini (Apioninae, Brentidae, Curculionoidea, Coleoptera) indicate an ancient co-diversification pattern of beetles and flowering plants. Mol. Phylogenet. Evol. 107: 179-190. https://dx.doi.org/10.1016/j. ympev.2016.10.015

Received: $11 / 06 / 2019$

Revised: 27/07/2019

Accepted: 20/08/2019

Published online: 10/10/2019 